\title{
Reduction of constrained systems with symmetries
}

Frans Cantrijn ${ }^{\text {a) }}$

Theoretical Mechanics Division, University of Gent, Krijgslaan 281, B-9000 Gent, Belgium

Manuel de León ${ }^{\text {b) }}$

Instituto de Matemáticas y Física Fundamental, CSIC, Serrano 123, E-28006 Madrid, Spain

Juan Carlos Marreroc)

Departamento de Matemática Fundamental, Facultad de Matemáticas, Universidad de la Laguna, Tenerife, Canary Islands, Spain

David Martín de Diego ${ }^{\text {d) }}$

Departamento de Economía Aplicada (Matemáticas), Facultad de CC. Economicas

y Empresariales, Avda Valle Esgueva 6, E-47011 Valladolid, Spain

(Received 13 July 1998; accepted for publication 21 September 1998)

A general model is proposed for constrained dynamical systems on a symplectic manifold which covers, among others, the description of Lagrangian and Hamiltonian systems with nonholonomic constraints and the canonical description of mechanical systems with a singular Lagrangian. The reduction properties of these systems in the presence of symmetry are investigated within this general framework. (C) 1999 American Institute of Physics. [S0022-2488(99)00902-0]

\section{INTRODUCTION}

In this paper, we propose a general model for constrained Hamiltonian systems on a symplectic manifold, providing a unified setting for the description of various types of mechanical systems with constraints. Special attention is then paid to aspects concerning symmetry and reduction for the class of systems under consideration.

The constraints encountered in classical mechanics can be classified, roughly speaking, into two different categories, which may be labeled as "internal constraints" and"external constraints," respectively. Internal constraints are those that find their origin in the degeneracy of the Lagrangian describing a certain system, which prevents a straightforward transition to an equivalent Hamiltonian formulation. This type of constraint usually reflects the presence of "gauge" degrees of freedom, and is, in fact, more relevant to relativistic mechanics and field theory. The standard treatment of degenerate (or singular) Lagrangian systems is based on the DiracBergmann constraint analysis (see, e.g., Ref. 1). An intrinsic geometric formulation and generalization of this theory is provided by the so-called presymplectic constraint algorithm, developed by Gotay and Nester. ${ }^{2-4}$ A different approach to singular Lagrangian systems, advocated by Tulczyjew, consists in treating them as implicit dynamical systems (see, e.g., Ref. 5).

External constraints refer to those physical constraints which are imposed on a given system from outside. Here we can make a further distinction between (time-independent or timedependent) holonomic and nonholonomic, one-sided and two-sided constraints. Holonomic constraints are restrictions on the admissible configurations (i.e., "positions") of the system under consideration, whereas nonholonomic constraints depend on the velocities in an essential way, i.e.,

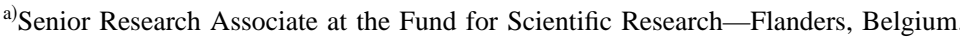

Electronic mail: frans.cantrijn@ rug.ac.be

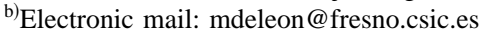

${ }^{\text {c) }}$ Electronic mail: jcmarrer@ull.es

${ }^{\mathrm{d})}$ Electronic mail: dmartin@esgueva.eco.uva.es
} 
they can not be integrated to relations depending on the position variables only. The prototype of nonholonomic constraints are the conditions for "rolling without slipping." For the purpose of this paper, we restrict our attention to the case of systems with two-sided, time-independent nonholonomic constraints. (For a geometric approach to systems with one-sided constraints, which are analytically expressed by inequalities, we refer to Refs. 6, 7.) The classical approach to nonholonomic mechanical systems is based on the method of Lagrange multipliers (see, e.g., Ref. 8 for a comprehensive treatment). The fundamental work of Vershik and Faddeev ${ }^{9,10}$ has marked the beginning of a period of intensive research on nonholonomic systems within the realm of geometric mechanics: see, for instance, Refs. 11-14 for a more detailed bibliography. In particular, the relevance of these studies for the further development of control theory has recently attracted a lot of attention (see, e.g., Refs. 15, 16, and references therein). We note, in passing, that nonholonomic systems have also been treated as implicit dynamical systems by Ibort et al. ${ }^{11}$

In spite of the difference in the "physical" nature of the constraints, it turns out that the geometrical models adopted for describing systems with either internal or external constraints, have many aspects in common. Indeed, in the canonical treatment of degenerate systems as well as in the Lagrangian and Hamiltonian treatment of nonholonomic systems, the search for consistent equations of motion eventually leads to a framework consisting of the following ingredients: a symplectic manifold $(P, \omega)$, a smooth function $H$ on $P$, a submanifold $M$ of $P$, and a distribution $F$ along $M$ (i.e., a subbundle of the restricted tangent bundle $T P P_{\mid M}$ ). Depending on the case, $P$ hereby represents the velocity phase space $T Q$ or the momentum phase space $T^{*} Q$ of the system under consideration, with underlying configuration space $Q$, and $\omega$ is either the PoincaréCartan 2-form on $T Q$, induced by a regular Lagrangian, or the canonical symplectic form on $T^{*} Q$. In the case of degenerate systems, $M$ is the "final constraint submanifold" generated by the appropriate constraint algorithm, and $F$ coincides either with $T M$ or with the tangent bundle of a larger submanifold containing $M$ (the primary constraint submanifold). $H$ denotes the energy function or the (extended) Hamiltonian. In the case of a nonholonomic system, $M$ simply denotes the constraint submanifold defined by the given external constraints, and the distribution $F$ is characterized by the property that its annihilator is the co-distribution generated by the reaction forces, induced by the constraints. The problem then consists in finding a vector field on $P$, generated by $H$, which is tangent to $M$ and compatible, in an appropriate sense, with the distribution $F$.

In the present paper we will take the above ingredients as building stones for constructing a general model for constrained dynamical systems in a symplectic setting. This model can be seen, in particular, as a unifying model for the description of degenerate systems as well as of mechanical systems with nonholonomic constraints. Our main goal then is to study the geometry of such systems in the presence of symmetry. Guided by various recent treatments of nonholonomic systems with symmetry (cf. Refs. 17, 15, 12, 18, 19), we will discuss in some detail the reduction problem for general constrained Hamiltonian systems with symmetry.

The scheme of this paper is as follows. In the next section we briefly recall some aspects of the geometrical approach to singular Lagrangian systems and to systems with nonholonomic constraints. In Sec. III we then propose a general model for constrained systems and investigate the existence and uniqueness conditions for the dynamics. In Sec. IV we deal with the problem of solving the dynamics. In Sec. V, we introduce symmetry into our model and present some general reduction results. After putting forward a classification of constrained systems with symmetry, inspired on the one introduced by Bloch et al. ${ }^{15}$ for nonholonomic systems, we describe some further reduction results for each class separately in Sec. VI. Finally, in Sec. VII we illustrate the obtained results on some particular cases.

Throughout this paper, we work in the category of smooth (i.e., $C^{\infty}$ ) objects. For convenience, we will usually not make a notational distinction between a (vector) bundle over a manifold and the ring of its smooth sections, i.e., if $F$ denotes a vector bundle over a manifold $N$ (for instance, a subbundle of $T N$ ), then $X \in F$ simply means that $X: N \rightarrow F$ is a section of $F$. The sole exception to this rule will be the occasional use of the notation $\mathfrak{X}(N)$ for the ring of smooth vector fields on $N$. 


\section{EQUATIONS OF MOTION OF CONSTRAINED LAGRANGIAN SYSTEMS}

Consider a smooth, finite dimensional manifold $Q$, with local coordinates denoted by $\left(q^{A}\right)$. As is well known, the tangent bundle $T Q$ of $Q$, with canonical projection $\tau_{Q}: T Q \rightarrow Q$, is equipped with a dilation vector field $\Delta$, i.e., the so-called Liouville vector field, and a canonical type $(1,1)$ tensor field $S$, called the vertical endomorphism, which determines the almost tangent structure of $T Q$. In the natural bundle coordinates $\left(q^{A}, v^{A}\right)$ of $T Q$ these objects read as

$$
\Delta=v^{A} \frac{\partial}{\partial v^{A}}, \quad S=\frac{\partial}{\partial v^{A}} \otimes d q^{A} .
$$

Given a Lagrangian on $T Q$, i.e., a smooth function $L: T Q \rightarrow \mathbb{R}$, one can define the corresponding Poincaré-Cartan 1- and 2-forms $\theta_{L}$ and $\omega_{L}$, respectively, and the energy function $E_{L}$, according to

$$
\theta_{L}=S^{*}(d L), \quad \omega_{L}=-d \theta_{L}, \quad E_{L}=\Delta(L)-L,
$$

with $S^{*}$ denoting the action of $S$ on 1-forms. In geometrical terms, the equations of motion for the Lagrangian system with Lagrangian $L$ can then be expressed by

$$
i_{Z} \omega_{L}=d E_{L}
$$

If $L$ is regular, that is, if the Hessian matrix $\left(\partial^{2} L / \partial v^{A} \partial v^{B}\right)$ is nondegenerate everywhere, then $\omega_{L}$ is a symplectic form. In that case (1) admits a unique solution for $Z$, which we will denote by $\Gamma_{L}$, and which is usually called the Euler-Lagrange vector field corresponding to $L$. In particular, $\Gamma_{L}$ is a second order differential equation field (SODE, for short), that is, $S\left(\Gamma_{L}\right)=\Delta$. The base integral curves $q^{A}(t)$ of $\Gamma_{L}$ (i.e., the projections of its integral curves onto $Q$ ) verify the EulerLagrange equations,

$$
\frac{d}{d t}\left(\frac{\partial L}{\partial \dot{q}^{A}}\right)-\frac{\partial L}{\partial q^{A}}=0
$$

For later use, we recall that the symplectic form $\omega_{L}$, corresponding to a regular Lagrangian, induces two bundle isomorphisms ("musical mappings") $b_{L}: T(T Q) \rightarrow T^{*}(T Q)$ and $\#_{L}: T^{*}(T Q) \rightarrow T(T Q)$, where $b_{L}(X)=i_{X} \omega_{L}$ and $\#_{L}=b_{L}^{-1}$.

\section{A. Singular Lagrangian systems}

A Lagrangian (system) is called singular, or degenerate, if the Hessian matrix $\left(\partial^{2} L / \partial v^{A} \partial v^{B}\right)$ is singular. In such a case, the equation of motion (1), in general, does not have a solution, and if a solution exists, it will not be unique. If the Poincaré-Cartan 2-form $\omega_{L}$ corresponding to a singular Lagrangian has constant rank and, hence, $\omega_{L}$ happens to be a presymplectic form, one can apply the so-called presymplectic constraint algorithm, developed by Gotay and Nester (see, e.g., Refs. 2, 3). This algorithm generates a descending sequence of constraint submanifolds which, under the appropriate conditions, converges to a closed immersed submanifold $P_{f}$ of $T Q$ (the "final constraint submanifold") on which there exist consistent equations of motion for the given system. More precisely, it follows by construction that the equation

$$
\left(i_{Z} \omega_{L}-d E_{L}\right)_{\mid P_{f}}=0
$$

admits at least one solution $Z$ which is everywhere tangent to $P_{f}$. In addition, one can always find a submanifold of $P_{f}$ on which there exists a unique solution $Z$ which also verifies the SODE condition (cf. Ref. 4). For quantization purposes, however, it is more convenient to develop the analysis of a degenerate Lagrangian system on an ambient symplectic space. This can be achieved by passing to an appropriate Hamiltonian formulation. 
Let us recall that, given a Lagrangian $L$, the Legendre map Leg: $T Q \rightarrow T^{*} Q$ is a fibered mapping over $Q$, which is locally written as

$$
\operatorname{Leg}\left(q^{A}, v^{A}\right)=\left(q^{A}, p_{A}\right)
$$

where $p_{A}=\partial L / \partial v^{A}$. If $\omega_{Q}$ denotes the canonical symplectic form on $T^{*} Q$, we have $\operatorname{Leg} * \omega_{Q}$ $=\omega_{L}$ (see, e.g., Ref. 20). Hence, $L$ is regular if and only if Leg is a local diffeomorphism, and $L$ is said to be hyperregular if Leg is a global diffeomorphism. For a singular Lagrangian system it is not possible, in general, to obtain a consistent Hamiltonian description. Let us assume, however, that $L$ is almost-regular, i.e., $M_{1}=\operatorname{Leg}(T Q)$ is a submanifold of $T^{*} Q$ and Leg is a submersion onto $M_{1}$ with connected fibers. In that case, the energy function $E_{L}$ projects onto a function $h_{1}: M_{1} \rightarrow \mathrm{R}$ which is uniquely determined by $h_{1} \circ \operatorname{Leg}_{1}=E_{L}$, where $\operatorname{Leg}_{1}$ simply stands for the "restriction" of Leg, regarded as a mapping from $T Q$ onto $M_{1}$. If we now denote by $\omega_{1}$ the pull-back of $\omega_{Q}$ to $M_{1}$, then the equation

$$
i_{X} \omega_{1}=d h_{1},
$$

is precisely the Hamiltonian counterpart of Eq. (1).

Starting from (4), one can again apply the presymplectic constraint algorithm which, in case the given problem is consistent, leads to a nonempty final constraint submanifold $M_{f}$ such that the equation

$$
\left(i_{X} \omega_{1}-d h_{1}\right)_{\mid M_{f}}=0
$$

admits well-defined solutions. This approach yields a global version of the classical DiracBergmann theory for constrained systems. ${ }^{1}$ Let " $\perp$ " denote the symplectic orthogonal with respect to the canonical symplectic form $\omega_{Q}$. Then, if $X$ is an arbitrary solution of (5), all other solutions will be of the form $X+Y$, with $Y \in T M_{f} \cap T M_{1}^{\perp}$.

A simple argument shows that, for almost regular Lagrangians, the Lagrangian and the Hamiltonian formulations are fully equivalent (see Refs. 2, 3). In particular, the final constraint submanifolds on both sides are connected via the Legendre transformation, in the sense that the latter induces a fibration $\operatorname{Leg}_{f}: P_{f} \rightarrow M_{f}$. Whenever $Z$ is a projectable solution of (3), its projection onto $M_{f}$ yields a solution of (5) and, conversely, given a solution $X$ of (5), any vector field $Z$ on $P_{f}$ which projects onto $X$ satisfies (3).

A geometric constraint algorithm, closely related to the Gotay-Nester approach, is the one developed by Hinds ${ }^{21}$ (see also Ref. 2 for a brief discussion). Again starting from (4), this algorithm generates a descending sequence of constraint submanifolds. In the favorable case, the algorithm stabilizes at a final constraint submanifold which, for simplicity, we will denote again by $M_{f}$. It is important to point out that, in general, this $M_{f}$ will be different from the final constraint submanifold obtained by the presymplectic constraint algorithm. In principle, both algorithms start to diverge from each other after the second step. This is due to the fact that in the Hinds algorithm, at each step, possibly new constraints are generated by imposing consistency conditions on the equations of motion induced on the previous constraint submanifold by a pullback procedure. In the presymplectic constraint algorithm, on the other hand, the consistency conditions are imposed on the equations obtained by taking the restriction of (4) to the successive constraint submanifolds. The equations of motion obtained through Hinds' algorithm can be written as

$$
i_{X} \omega_{f}=d h_{f},
$$

with $\omega_{f}$ and $h_{f}$ denoting the pull-back to $M_{f}$ of $\omega_{1}$ and $h_{1}$, respectively. Given a solution $X$ of this equation, it follows that $X+Y$ is also a solution for any $Y \in T M_{f} \cap T M_{f}^{\perp}$. Note that (6) is an equation induced on the final constraint submanifold, i.e., it expresses an equality of 1-forms on 
$M_{f}$, whereas (5) represents an equality of 1-forms on $M_{1}$, restricted to points of the corresponding $M_{f}$. This indeed reflects the difference in spirit between both algorithms, as described above.

Following Dirac, ${ }^{1}$ the constraints produced in the course of the constraint analysis can be classified in two different ways. On the one hand, depending on the order of appearance, there are primary, secondary, (tertiary, etc.,) constraints. On the other hand, there is the more significant distinction between first and second class constraints. In physics, it is customary to assume that all first class constraints (primary, secondary,...) are generators of gauge transformations, i.e., transformations that do not change the physical state of the system (see, e.g., Refs. 22, 23). This property is automatically verified when applying Hinds' algorithm. In the Gotay-Nester approach, all primary first class constraints generate gauge transformations but, in general, this need not be the case for all subsequent (secondary,...) first class constraints (see Ref. 2 for more details). From a physical point of view, therefore, it may be argued that (6) is in better agreement with the "standard" interpretation of gauge transformations than (5).

We will now recast the equations (5) and (6) into a form which better serves our purpose. Taking an arbitrary extension $H_{1}: T^{*} Q \rightarrow \mathbb{R}$ of the Hamiltonian $h_{1}: M_{1} \rightarrow \mathbb{R}$, it follows that (5) is formally equivalent to

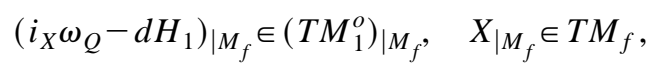

where $T M_{1}^{o}$ is the annihilator of $T M_{1}$ in $T^{*} T^{*} Q$. Locally, these conditions precisely generate the equations of motion ensuing from the classical Dirac-Bergmann constraint analysis. Likewise, taking an arbitrary extension $H_{f}$ of $h_{f}$ to $T^{*} Q$, (6) can be rewritten in terms of the canonical symplectic form as follows:

$$
\left(i_{X} \omega_{Q}-d H_{f}\right)_{\mid M_{f}} \in T M_{f}^{o}, \quad X_{\mid M_{f}} \in T M_{f},
$$

where it should be emphasized again that, for the same system, the constraint submanifolds $M_{f}$ in (7) and (8), in general, need not be the same.

\section{B. Nonholonomic Lagrangian systems}

In this section, we start by considering a regular Lagrangian system with Lagrangian $L: T Q$ $\rightarrow \mathbb{R}$, subjected to a set of nonholonomic constraints which are linear in the velocities, i.e., they can be (locally) represented by a set of independent functions of the form $\phi_{i}:=\mu_{i A}(q) v^{A}$, for 1 $\leqslant i \leqslant m$. We can describe this nonholonomic Lagrangian system in geometrical terms as follows. The constraint equations $\phi_{i}=0$ define a $(n-m)$-dimensional distribution $\mathcal{D}$ on the $n$-dimensional configuration manifold $Q$. We denote its total space by $D$, which is a $(2 n-m)$-dimensional submanifold of $T Q$ : the constraint submanifold. For simplicity we always assume in the sequel that $\tau_{Q}(D)=Q$, i.e., the constraints are "purely kinematical" in the sense that they do not impose restrictions on the allowable positions. The motions of the system are forced to take place on $D$, and this requires the introduction of some (unknown) "reaction forces." In de León et al., ${ }^{24}$ an intrinsic expression for the equations of motion was obtained, which we will describe below.

First of all, we define a distribution $\mathcal{D}^{v}$ on $T Q$ by prescribing its annihilator as a subbundle of $T^{*} T Q$ which, along the constraint submanifold $D$, represents the bundle of reaction forces. More precisely, given a set of independent 1 -forms $\left\{\mu_{i} ; 1 \leqslant i \leqslant m\right\}$ on $Q$, which locally generate the annihilator $\mathcal{D}^{o}$ of $\mathcal{D}$, we put

$$
\left(\mathcal{D}^{v}\right)^{o}=\left\langle\mu_{i}^{v}\right\rangle,
$$

where $\mu_{i}^{v}$ denotes the vertical lift of the 1 -form $\mu_{i}$ to $T Q$ (see Ref. 20). A direct computation reveals that $\mathcal{D}^{v}$ is, in fact, globally defined. Note, in passing, that with $\mu_{i}=\mu_{i A} d q^{A}$, the given constraint functions $\phi_{i}$ are precisely the evaluation maps of these 1-forms.

Next, it can then be shown that the equations of motion for such a nonholonomic mechanical system are given by 


$$
\left(i_{X} \omega_{L}-d E_{L}\right)_{\mid D} \in\left(\mathcal{D}^{v}\right)^{o}, \quad X_{\mid D} \in T D
$$

It should be pointed out that each solution of (9) (if there exists one) is automatically a SODE along $D$. This implies that, in local coordinates, the integral curves of $X$ on $D$ are of the form $\left(q^{A}(t), \dot{q}^{A}(t)\right)$, whereby the $q^{A}(t)$ are solutions of the system of differential equations

$$
\frac{d}{d t}\left(\frac{\partial L}{\partial \dot{q}^{A}}\right)-\frac{\partial L}{\partial q^{A}}=\lambda^{i} \mu_{i A},
$$

together with the constraint equations $\mu_{i A}(q) \dot{q}^{A}=0$, and where the $\lambda^{i}$ are Lagrange multipliers.

We will now describe a procedure which permits us to decide under what conditions (9) admits a solution and, if these conditions are fulfilled, to obtain a solution by projection of the Euler-Lagrange vector field of the corresponding unconstrained system.

Applying the isomorphism $\#_{L}$ to the co-distribution $\left(\mathcal{D}^{v}\right)^{o}$ we obtain the symplectic orthogonal complement (with respect to $\omega_{L}$ ) of $\mathcal{D}^{v}$, i.e., $\sharp_{L}\left(\left(\mathcal{D}^{v}\right)^{o}\right)=\left(\mathcal{D}^{v}\right)^{\perp}$. Obviously, $\operatorname{dim}\left(\mathcal{D}^{v}\right)^{\perp}$ is equal to the number of independent constraints. We will say that the given nonholonomic system satisfies the compatibility condition if $T_{x} D \cap\left(\mathcal{D}_{x}^{v}\right)^{\perp}=\{0\}$ at each point $x \in D$. In such a case, taking into account that $\operatorname{dim}\left(\mathcal{D}_{x}^{v}\right)^{\perp}=m$, we have a direct sum decomposition,

$$
T_{x}(T Q)=T_{x} D \oplus\left(\mathcal{D}_{x}^{v}\right)^{\perp}, \quad x \in D,
$$

which, in turn, gives rise to two complementary projectors, say

$$
\mathcal{P}_{x}: T_{x}(T Q) \rightarrow T_{x} D, \quad \mathcal{Q}_{x}: T_{x}(T Q) \rightarrow\left(\mathcal{D}_{x}^{v}\right)^{\perp}
$$

A direct calculation shows that $\Gamma_{L, D}=\mathcal{P}\left(\Gamma_{L_{\mid D}}\right)$ is a solution of (9). Moreover, one can easily show that this solution is necessarily unique. ${ }^{24}$ The procedure just described is essentially equivalent to the classical one based on the use of Lagrange multipliers.

If the nonholonomic system does not verify the compatibility condition, that is, $T_{x} D \cap\left(\mathcal{D}_{x}^{v}\right)^{\perp} \neq\{0\}$ at some points $x \in D$, we can develop a constraint algorithm which is very similar to the one described above for singular Lagrangians (cf. Ref. 24). Under the appropriate conditions, this algorithm determines a final constraint submanifold $D_{f}$ on which there exist consistent equations of motion for the given constrained problem. More precisely, the algorithm guarantees the existence of well-defined solutions $X$ of the system

$$
\left(i_{X} \omega_{L}-d E_{L}\right)_{\mid D_{f}} \in\left(\mathcal{D}^{v}\right)^{o}, \quad X_{\mid D_{f}} \in T D_{f}
$$

(see Ref. 24 for details). Again, it turns out that a solution of (11) is a SODE along $D_{f}$.

The previous analysis of nonholonomic systems can be further extended to the case where, in addition, the Lagrangian happens to be singular (see Refs. 25, 26). To fix the ideas, let us assume that $L$ is almost regular, and that $\operatorname{ker}(\operatorname{Leg})_{*} \subset \mathcal{D}^{c}$, where $\mathcal{D}^{c}$ denotes the tangent or complete lift of $\mathcal{D}$ to $T Q$, i.e., $\mathcal{D}^{c}$ is the distribution on $T Q$ whose annihilator is given by $\left(\mathcal{D}^{c}\right)^{o}=\left\langle\mu_{i}^{v}, \mu_{i}^{c}\right\rangle$. The nonholonomic mechanical system $(L, D)$ is then also said to be almost regular. Under these assumptions, the following is proved in Ref. 25 (using the notations of the previous subsection):

- $\bar{D}=\operatorname{Leg}(D)$ is a submanifold of $M_{1}=\operatorname{Leg}(T Q)$, and the restriction $\operatorname{Leg}_{\mid D}: D \rightarrow \bar{D}$ is a surjective submersion whose fiber at a point $x \in \bar{D}$ is precisely given by $\operatorname{Leg}^{-1}(x)$.

- If $\left\{\mu_{i}\right\}$ is a basis of $\mathcal{D}^{o}$, then $\left(\mathcal{D}^{\bar{v}}\right)^{o}=\left\langle\pi_{Q}^{*} \mu_{i}\right\rangle$ defines a distribution $\overline{\mathcal{D}}^{\bar{v}}$ on $T^{*} Q$, where $\pi_{Q}: T^{*} Q \rightarrow Q$ is the canonical projection. By construction, the distributions $\mathcal{D}^{v}$ and $\overline{\mathcal{D}}^{\bar{v}}$ are Leg-related.

- Let $\overline{\mathcal{D}}_{1}^{\bar{v}}$ be the distribution on $M_{1}$, the annihilator of which is the co-distribution obtained by taking the pull-back to $M_{1}$ of the forms generating $\left(\overline{\mathcal{D}}^{\bar{v}}\right)^{o}$. The system 


$$
\left(i_{Y} \omega_{1}-d h_{1}\right)_{\mid \bar{D}} \in\left(\overline{\mathcal{D}}_{1}^{\bar{v}}\right)^{o}, \quad Y_{\mid \bar{D}} \in T \bar{D},
$$

is then equivalent to the system

$$
\left(i_{X} \omega_{L}-d E_{L}\right)_{\mid D} \in\left(\mathcal{D}^{v}\right)^{o}, \quad X_{\mid D} \in T D
$$

where $h_{1}$ is the projection of $E_{L}$ onto $M_{1}$. Indeed, one can develop a constraint algorithm for both systems such that, at each stage, the respective constraint submanifolds $D_{k}$ and $\bar{D}_{k}$ are Leg-related, that is, $\operatorname{Leg}\left(D_{k}\right)=\bar{D}_{k}$. Moreover, with $D_{f}$ and $\bar{D}_{f}$ denoting the final constraint submanifolds, one can show that the Legendre map induces a surjective submersion $\operatorname{Leg}_{f}: D_{f} \longrightarrow \bar{D}_{f}$ which projects solutions onto solutions.

In particular, when applying the constraint algorithm to (12) we end up with the dynamical equation,

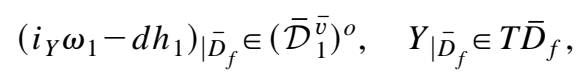

which, by construction, admits well-defined solutions $Y$. Finally, as in the treatment of (free) singular Lagrangian systems, discussed in the previous subsection, one can prove the formal equivalence of (13) with

$$
\left(i_{Y} \omega_{Q}-d H\right)_{\mid \bar{D}_{f}} \in\left(\overline{\mathcal{D}}^{\bar{v}} \cap T M_{1}\right)^{o}, \quad Y_{\mid \bar{D}_{f} \in T \bar{D}_{f}}
$$

where $H: T^{*} Q \rightarrow \mathrm{R}$ is an arbitrary extension of $h_{1}$.

Remark II.1: In the previous discussion we have confined ourselves to the case of linear nonholonomic constraints. Much of the above, however, applies equally well to the case of affine or even nonlinear constraints. For instance, for a regular Lagrangian system, subjected to nonlinear, nonholonomic constraints, described by a submanifold $M$ of the tangent bundle $T Q$, the equations of motion are again of the form (9), with the vector subbundle $D$ being replaced by $M$ and $\left(\mathcal{D}^{v}\right)^{o}$ by the co-distribution $S^{*}\left((T M)^{\circ}\right)$ (see, e.g., Refs. 27, 28).

\section{A GENERAL FRAMEWORK FOR CONSTRAINED SYSTEMS}

When looking at the systems (7), (8), (9), (11) and (14), we see that, in spite of the difference in (physical) origin and interpretation, they all have a similar geometrical structure. This prompts us to introduce the following general model for constrained dynamical systems within a symplectic setting.

Consider a symplectic manifold $(P, \omega)$, a smooth function $H: P \rightarrow \mathrm{R}$ (the Hamiltonian), an embedded submanifold $M$ of $P$ (the constraint submanifold) and a distribution $F$ on $P$ along $M$, i.e., $F$ is a vector subbundle of $T P_{\mid M}$. We are then interested in the following problem: find a smooth section $X$ of the restricted tangent bundle $T P P_{\mid M} \rightarrow M$, such that

$$
\left(i_{X} \omega-d H\right)_{\mid M} \in F^{o}, \quad X \in T M
$$

with $F^{o}$ the annihilator of $F$ in $T^{*} P_{\mid M}$. In particular, $X$ then defines a vector field on $M$. It is clear that $(7),(8),(9),(11)$ and (14) belong to the class of problems described by (15). (We thereby ignore the technicality that in the treatment of singular Lagrangian systems, the final constraint submanifold, in principle, may be an immersed rather than an embedded submanifold.)

In what follows we will denote by $b: T P \rightarrow T^{*} P, X \mapsto i_{X} \omega$ and $\sharp=b^{-1}: T^{*} P \rightarrow T P$, the bundle isomorphisms over $P$ induced by the symplectic form $\omega$.

We now first study the problem of the existence and uniqueness of solutions of the constrained system (15).

Proposition III.1: (i) (Existence) The system (15) admits a solution if and only if 


$$
d H(x) \in\left(F_{x} \cap T_{x} M^{\perp}\right)^{o},
$$

at each point $x \in M$.

(ii) (Uniqueness) If (15) has a solution, then it is unique if and only if

$$
F^{\perp} \cap T M=0 .
$$

Proof: (i) If $X$ is a solution of (15) then, since $X(M) \subset T M$, we have $i_{X} \omega_{\mid M} \in\left(T M^{\perp}\right)^{o}$, from which it follows that

$$
d H_{\mid M} \in F^{o}+\left(T M^{\perp}\right)^{o}=\left(F \cap T M^{\perp}\right)^{o} .
$$

Conversely, assume that $d H_{\mid M} \in F^{o}+\left(T M^{\perp}\right)^{o}$. Then, $d H_{\mid M}-\beta \in\left(T M^{\perp}\right)^{o}$ for some $\beta \in F^{o}$. Since $b(T M)=\left(T M^{\perp}\right)^{o}$, we deduce that there exists a vector field $X$ satisfying (15).

(ii) Now, let $X$ and $X^{\prime}$ be two solutions of (15). Then

$$
X-X^{\prime} \in F^{\perp} \cap T M .
$$

Hence, if a solution exists, it will be unique if and only if $F^{\perp} \cap T M=0$.

Note that the existence condition can be equivalently expressed as

$$
X_{H \mid M} \in T M+F^{\perp},
$$

where $X_{H}$ denotes the (unconstrained) Hamiltonian vector field on $(P, \omega)$ with Hamiltonian $H$. Hence, any solution $X$ of (15) is of the form

$$
X=X_{H \mid M}+Z,
$$

for some $Z \in F^{\perp}$. An interesting special case occurs when $\operatorname{rank} F=\operatorname{dim} M$ or, equivalently, $\operatorname{dim} F_{x}=\operatorname{dim} T_{x} M$ for all $x \in M$.

Corollary III.2: If rank $F=\operatorname{dim} M$, then the condition $F^{\perp} \cap T M=0$ implies both the existence and uniqueness of a solution of (15).

Proof: A simple algebraic argument shows that, under the given assumptions, $T P_{\mid M}=F^{\perp}$ $\oplus T M$. Taking the symplectic complements of both sides, we find that $0=F \cap T M^{\perp}$ and, hence, $T^{*} P_{\mid M}=\left(F \cap T M^{\perp}\right)^{o}$. The result now readily follows from the previous Proposition. Q.E.D.

Under the conditions of the Corollary, (15) is a constrained Hamiltonian system in the sense of Marle, ${ }^{19}$ who has studied such systems in the more general setting of Poisson manifolds.

Let us now check the existence and uniqueness conditions for the examples discussed in the previous section. For the nonholonomic system (9), with a regular Lagrangian, we have $(P, \omega)$ $=\left(T Q, \omega_{L}\right), M=D$ and $F=\mathcal{D}^{v}$. The compatibility condition introduced for such a system precisely coincides with the unicity condition from Proposition 3.1. Since a simple counting of dimensions shows that rank $\mathcal{D}^{v}=\operatorname{dim} D$, it follows from the above Corollary that a compatible nonholonomic system indeed admits a unique solution. For the other cases (7), (8), (11) and (14), we note that the equations of motion are obtained after applying a constraint algorithm. The latter is precisely conceived so as to guarantee the existence of a consistent solution, i.e., in these cases the existence condition of Proposition 3.1 holds by construction. The uniqueness condition, however, need not be satisfied: in general, there will be "gauge degrees of freedom."

Returning to the general model (15), it is important to point out that if the system admits a solution $X$, it need not be true, in general, that (the restriction of) $H$ is a first integral of $X$. In classical mechanics, for instance, it is well known that imposing nonholonomic constraints on a conservative mechanical system may destroy the conservation of energy (see, e.g., Ref. 19). An additional assumption on the nature of the constraints therefore is needed to ensure the conservation of energy. For a Lagrangian system subject to general (i.e., not necessarily linear) nonholonomic constraints, a sufficient condition for the energy $E_{L}$ to be conserved is that the constraints are "homogeneous," which, in geometrical terms, means that the dilation vector field $\Delta$ should be 
tangent to the constraint submanifold (see Refs. 27, 28, where the less appropriate denomination "ideal constraints" was used instead of homogeneous constraints). In the case of linear constraints, this condition is always fulfilled.

Remark III.3: If (15) admits no solution, then it is possible to develop a constraint algorithm which, at least in case the given problem is consistent, will lead to a final constraint submanifold $M_{f}$ on which there exist a well-defined dynamics. The system to be considered then reads as

$$
\left(i_{X} \omega-d H\right)_{\mid M_{f}} \in F^{o}, \quad X_{\mid M_{f}} \in T M_{f},
$$

which is again of the same type as (15). By construction this system now has well-defined solutions. Therefore, without loss of generality, we will henceforth always assume that the existence condition of Proposition 3.1 is satisfied

\section{SOLVING THE DYNAMICS}

Given a constrained system of the form (15) for which condition (i) of Proposition 3.1 holds, we will now indicate how one can explicitly construct a (local) solution for the dynamics.

Let $X_{H}$ again denote the "unconstrained" Hamiltonian system on $(P, \omega)$, corresponding to the Hamiltonian $H$. Take a local basis $\left\{\mu_{i} ; 1 \leqslant i \leqslant m\right\}$ of $F^{o}$, and let $\left\{\Phi_{a} ; 1 \leqslant a \leqslant s\right\}$ be an independent set of constraint functions which locally define $M$. Denote by $Z_{i}$ the symplectic gradient of $\mu_{i}$, that is, $b\left(Z_{i}\right)=\mu_{i}$. Then, $F^{\perp}$ is locally generated by the vector fields $Z_{i}$ and, according to (16), any solution $X$ of (15) can be written as

$$
X=X_{H}+\lambda^{i} Z_{i}
$$

where the $\lambda^{i}$ are Lagrange multipliers which can be determined from the tangency condition:

$$
0=X\left(\Phi_{a}\right)_{\mid M}=X_{H}\left(\Phi_{a}\right)_{\mid M}+\lambda^{i} Z_{i}\left(\Phi_{a}\right)_{\mid M}, \quad \forall a .
$$

Indeed, the existence condition for solutions of (15), in particular, implies that this system of equations can be solved for the $\lambda^{i}$, i.e., on $M$ we have

$$
\operatorname{rank}\left(Z_{i}\left(\Phi_{a}\right)\right)=\operatorname{rank}\left(Z_{i}\left(\Phi_{a}\right) ;-X_{H}\left(\Phi_{a}\right)\right) .
$$

Of course, the solution for the $\lambda^{i}$ need not be unique.

Next, let us assume that both conditions of Proposition 3.1 are satisfied, so that the system admits a unique solution. Our goal now is to construct a projection operator which allows us to deduce the constrained dynamics from the unconstrained dynamics $X_{H}$.

From the assumption $F^{\perp} \cap T M=0$ it readily follows that for each $x \in M, \operatorname{dim} F_{x}^{\perp}$ $\leqslant$ codim $T_{x} M$, i.e., $m=$ corank $F \leqslant \operatorname{codim} M=s$. We can now distinguish the following two cases.

Assume $m=s$.

A simple dimensional argument shows that

$$
T P_{\mid M}=T M \oplus F^{\perp}
$$

Therefore, there exist two complementary projectors $\mathcal{P}: T P_{\mid M} \rightarrow T M$ and $\mathcal{Q}: T P{ }_{\mid M} \rightarrow F^{\perp}$ and it is straightforward to check that $\mathcal{P}\left(X_{H}\right)$ is a solution of (15). Using the above notations, a local expression for $\mathcal{P}$ is given by

$$
\mathcal{P}=I d-\mathcal{C}^{i j} Z_{i} \otimes d \Phi_{j},
$$

where $\left(\mathcal{C}^{i j}\right)$ is the inverse of the regular matrix $\left(\mathcal{C}_{i j}\right)$, with $\mathcal{C}_{i j}=Z_{j}\left(\Phi_{i}\right)$. Hence we obtain

$$
\mathcal{P}\left(X_{H}\right)=X_{H}-\mathcal{C}^{i j} X_{H}\left(\Phi_{j}\right) Z_{i}
$$


Assume $m<s$.

In this case, we have

$$
T M \oplus F^{\perp} \varsubsetneqq T P_{\mid M},
$$

with two complementary projectors $\mathcal{P}: T M \oplus F^{\perp} \rightarrow T M$ and $\mathcal{Q}: T M \oplus F^{\perp} \rightarrow F^{\perp}$. From the existence condition it easily follows that $X_{H \mid M} \in T M \oplus F^{\perp}$. As above, the projection $\mathcal{P}\left(X_{H}\right)$ then provides the unique solution of the constrained dynamics.

The matrices $\left(Z_{i}\left(\Phi_{a}\right)\right)$ and $\left(Z_{i}\left(\Phi_{a}\right) ;-X_{H}\left(\Phi_{a}\right)\right)$, with $(1 \leqslant i \leqslant m ; 1 \leqslant a \leqslant s)$, both have maximal rank $m$. To obtain an explicit (local) description for $\mathcal{P}\left(X_{H}\right)$ we only need to select $m$ independent rows from the matrix $\left(Z_{i}\left(\Phi_{a}\right)\right)$. Without loss of generality, we may assume these to be the first $m$ rows $(1 \leqslant a \leqslant m)$, so that we recover (18).

Remark IV.1: Recently, various authors have pointed out that the dynamics of nonholonomic systems can be conveniently described in terms of a "pseudo-Poisson' bracket (see, e.g., Refs. $18,29,30)$. On the other hand, in Refs. 31, 25, a unified treatment of constrained systems has also been proposed in terms of Dirac brackets. The relation between these various bracket approaches has been discussed in Cantrijn et al. ${ }^{32}$ It is rather straightforward to see that these bracket formulations of constrained dynamics can be extended to the general model for constrained systems considered in this paper, but we will not further enter into this matter here.

In the next three sections we wish to investigate the effect of symmetry on the dynamics of constrained systems of type (15). In particular, we will describe various reduction schemes for such systems. The subsequent analysis remains close in spirit to some related treatments of nonholonomic systems with symmetry (see, for instance, Refs. 33, 17, 15, 27, 34, 19, 35).

\section{SYMMETRY AND REDUCTION}

Consider a constrained system of the form (15) and let there be a given symplectic action $\Phi: G \times P \rightarrow P$ of a Lie group $G$ on the symplectic manifold $(P, \omega)$, such that the submanifold $M$, the Hamiltonian function $H$ and the vector subbundle $F$ are $G$-invariant. For simplicity we will always assume that this action is free and proper. For each $g \in G$ and $x \in P$ we put $\Phi(g, x)$ $=\Phi_{g}(x)=g x$. The infinitesimal generator (fundamental vector field) corresponding to $\xi \in \mathfrak{g}$, with $\mathfrak{g}$ the Lie algebra of $G$, will be denoted by $\xi_{P}$. By assumption we thus have for all $g \in G$,

- $\Phi_{g}^{*}(H)=H \circ \Phi_{g}=H$;

- $\Phi_{g}(M) \subseteq M$;

- $T \Phi_{g}\left(F_{x}\right)=F_{\Phi_{g}(x)}$, for all $x \in M$.

If (15) admits a solution $X$, it is routine to verify that $\Phi_{g}^{*} X$ will also be a solution for each $g \in G$. This still means that at each point $x \in M, \Phi_{g}^{*} X(x)-X(x) \in F_{x}^{\perp} \cap T_{x} M$. In particular, in case (15) has a unique solution, the latter will be $G$-invariant.

In discussing the reduction of a $G$-invariant solution of (15) we will proceed in two stages. First, we will show that the above assumptions already allow us to construct a Poisson reduction. Next, upon invoking an additional hypothesis, we will establish a kind of symplectic reduction, in the sense of the one derived by Bates and Śniatycki ${ }^{17}$ for nonholonomic Hamiltonian systems.

(i) Poisson reduction. Since the action $\Phi$ is free and proper, the orbit space $\bar{P}=P / G$ is a differentiable manifold and $\rho: P \rightarrow \bar{P}$ is a principal bundle over $\bar{P}$ with structure group $G$, whereby $\rho$ denotes the natural projection. Moreover, $\Phi$ being a symplectic action, it is, in particular, a Poisson action with respect to the natural Poisson structure induced by $\omega$ on $P$, i.e., it leaves the corresponding Poisson tensor field $\Lambda$ on $P$ invariant. It is known that the orbit space $\bar{P}$ then admits a unique Poisson structure such that the projection $\rho$ becomes a Poisson map (see, e.g., Ref. 36). The corresponding Poisson tensor field $\bar{\Lambda}$ on $\bar{P}$ is unambiguously determined by

$$
\bar{\Lambda}(d \bar{f}, d \bar{g})(\bar{y})=\Lambda\left(\rho^{*} d \bar{f}, \rho^{*} d \bar{g}\right)(y),
$$


for all $\bar{f}, \bar{g} \in C^{\infty}(\bar{P})$ and $y \in \rho^{-1}(\bar{y})$. Let $\overline{\#}: T^{*} \bar{P} \rightarrow T \bar{P}$ be the linear bundle map induced by $\bar{\Lambda}$ according to

$$
\langle \#(\bar{\alpha}), \bar{\beta}\rangle=\bar{\Lambda}_{\bar{y}}(\bar{\alpha}, \bar{\beta}),
$$

for all $\bar{y} \in \bar{P}$ and $\bar{\alpha}, \bar{\beta} \in T_{\bar{y}}^{*} \bar{P}$.

The Hamiltonian $H$ being $G$-invariant, it induces a function $\bar{H}$ on $\bar{P}$. Moreover, $M$ is also assumed to be $G$-invariant and, clearly, the $G$-action induced by $\Phi$ on $M$ will still be free and proper. Thus, the quotient manifold $\bar{M}=M / G$ is a smooth submanifold of $\bar{P}$. Finally, we note that the $G$-invariance of $F$ also implies the $G$-invariance of $F^{\perp}$. For each $\bar{x} \in \bar{M}$ we put $\left(\overline{F^{\perp}}\right)_{\bar{x}}$ $=T \rho\left(F_{x}^{\perp}\right)$ for some $x \in \rho^{-1}(\bar{x})(\subset M)$. This definition is independent of the choice of $x$ $\in \rho^{-1}(\bar{x})$. We then put

$$
\overline{F^{\perp}}=\cup_{\bar{x} \in \bar{M}}\left(\overline{F^{\perp}}\right)_{\bar{x}}
$$

which defines a generalized distribution on $\bar{P}$ along $\bar{M}$. In principle, the bundle $\overline{F^{\perp}}$ need not have constant rank. Assume now that there exists a $G$-invariant solution $X$ of (15). As pointed out above, this will automatically be the case if the equation admits a unique solution. Then, $X$ is projectable onto $\bar{M}$ and its projection $\bar{X}$ verifies

$$
\bar{X} \in \overline{\#}(d \bar{H})+\overline{F^{\perp}},
$$

that is,

$$
\bar{X}=X_{\bar{H} \mid \bar{M}}+\bar{Z},
$$

for some $\bar{Z} \in \overline{F^{\perp}}$, with $X_{\bar{H}}=\#(d \bar{H})$. Indeed, according to (16) we can always write $X$ in the form $X=X_{H}+Z$, with $Z \in F^{\perp}$. The symmetry assumptions already guarantee the projectability of the Hamiltonian vector field $X_{H}$. Therefore, if $X$ is $G$-invariant, $Z$ is also $G$-invariant and its projection onto $\bar{M}$ is a section of $\overline{F^{\perp}}$.

Next, we will show that under an additional condition, the reduced dynamics $\bar{X}$ can be expressed in terms of a 2-form defined on a vector subbundle of $T \bar{P}_{\mid \bar{M}}$. The analysis closely follows the one developed in Ref. 17 (see also Ref. 34).

(ii) Bates-Sniatycki reduction. In what follows, we assume that there exists a $G$-invariant solution $X$ of (15) such that $X \in F$. Recall that the latter assumption, in particular, implies that $X(H)=0$.

Remark V.1: For the mechanical systems considered in Sec. II, the condition that the constrained dynamics should belong to the distribution $F$ is not at all restrictive. Indeed, for (7) and (8) we have that every solution $X$ automatically belongs to $F$ since, in those cases, $T M \subset F$. In the case of (9) and (11), the property that $X \in F$ is a consequence of the fact that $X$ is a SODE. Finally, for (14), the condition will be satisfied if the solution $Y$ on $\bar{D}_{f}$ is the projection of a SODE along a submanifold of $D_{f}$. It is known that one can always find such a submanifold and such a solution. ${ }^{25}$

In the sequel, we will denote by $\mathcal{V}$ the subbundle of $T P$ whose fibers are the tangent spaces to the $G$-orbits, i.e., $\mathcal{V}_{x}=T_{x}(G x)$ or, equivalently, $\mathcal{V}=\operatorname{ker} T \rho$. Note that $\mathcal{V}_{x} \subset T_{x} M$ for all $x \in M$, i.e., $\mathcal{V}_{\mid M} \subset T M$. For simplicity, we will also usually write $\mathcal{V}$, instead of $\mathcal{V}_{\mid M}$, when referring to its restriction to $M$ (the precise meaning should be clear from the context).

We now define a (generalized) vector subbundle $U$ of $T P_{\mid M}$, whose fiber at $x \in M$ is given by

$$
U_{x}=\left\{v \in F_{x} \cap T_{x} M / \omega(v, \tilde{\xi})=0, \text { for all } \tilde{\xi} \in \mathcal{V}_{x} \cap F_{x}\right\}
$$


In general, this bundle need not be of constant rank, i.e., it determines a generalized distribution on $P$ along $M$. In the sequel, however, we will always tacitly assume that $U$ is a genuine vector bundle over $M$, although much of the analysis also holds in the more general situation. Note that $U=(F \cap T M) \cap(\mathcal{V} \cap F)^{\perp}$, where $(\mathcal{V} \cap F)^{\perp}$ is the $\omega$-complement of $\mathcal{V} \cap F$ in $T P_{\mid M}$. It is readily seen that $U$ is $G$-invariant and, hence, projects onto a subbundle $\bar{U}$ of $T \bar{P}_{\mid \bar{M}}$. Let us now denote by $\omega_{U}$ the restriction of $\omega$ to $U$. Clearly, $\omega_{U}$ is also $G$-invariant and since, moreover, $i \tilde{\xi} \omega_{U}=0$ for all $\widetilde{\xi} \in \mathcal{V} \cap U$, the 2-form $\omega_{U}$ pushes down to a 2 -form $\omega_{\bar{U}}$ on $\bar{U}$ (i.e., $\omega_{\bar{U}}$ only acts on vectors belonging to $\bar{U})$. Similarly, the restriction of $d H$ to $U$, denoted by $d_{U} H$, pushes down to a 1 -form $d_{\bar{U}} \bar{H}$ on $\bar{U}$, which is simply the restriction of $d \bar{H}$ to $\bar{U}$. Note that neither $\omega_{\bar{U}}$ nor $d_{\bar{U}} \bar{H}$ are genuine differential forms on $\bar{M}$; they are exterior forms on a vector bundle over $\bar{M}$, with smooth dependence on the base point.

Proposition V.2: Let $X$ be a G-invariant solution of (15) such that, in addition, $X$ belongs to $F$. Then, the projection $\bar{X}$ of $X$ onto $\bar{M}$ is a section of $\bar{U}$ satisfying the equation

$$
i_{\bar{X}} \omega_{\bar{U}}=d_{\bar{U}} \bar{H}
$$

Proof: Essentially, all that remains to be checked is that $X$ is a section of $U$. Along $M$, the given solution $X$ verifies

$$
i_{X} \omega=d H+\beta,
$$

with $\beta \in F^{o} . H$ being $G$-invariant, it follows that for any section $\widetilde{\xi}$ of $\mathcal{V} \cap F, d H(\widetilde{\xi})=0$. Since, obviously, we also have $\beta(\widetilde{\xi})=0$, we may indeed conclude that $X \in U$. Consequently, the following relation holds along $M$ :

$$
i_{X} \omega_{U}=d_{U} H
$$

The remainder of the proof now readily follows from the symmetry assumptions and from the previous considerations.

Q.E.D.

It is important to observe that, in general, the 2 -form $\omega_{U}^{-}$may be degenerate. However, in the case of a mechanical system with linear nonholonomic constraints, for instance, one can prove that $\omega_{\bar{U}}^{-}$is nondegenerate, such that $\left(\bar{U}, \omega_{\bar{U}}\right)$ becomes a symplectic vector bundle over $\bar{M}$ (see Ref. 17). The reduced dynamics is then uniquely determined by the equation mentioned in the previous Proposition.

In the next section, we will identify three distinguished classes of constrained systems with symmetry, which will be analyzed in some more detail.

\section{A CLASSIFICATION OF CONSTRAINED SYSTEMS WITH SYMMETRY}

We again consider a constrained system (15) with symmetry, as described in the previous section. Recall that $\mathcal{V}=\operatorname{ker} T \rho$. For each infinitesimal generator $\xi_{P}$ of the given group action on $P$, corresponding to some $\xi \in \mathfrak{g}$, the restriction to $M$ is precisely the infinitesimal generator $\xi_{M}$ of the induced action on $M$. If $\xi_{M}$ is a section of $\mathcal{V} \cap F$, we will call it a horizontal symmetry of the given constrained system (see also Refs. 17, 15). The following classification, which is inspired on the one introduced by Bloch et al. ${ }^{15}$ for mechanical systems with linear or affine nonholonomic constraints, reflects the various possible ways the subspaces $\mathcal{V}_{x}$ and $F_{x}$ may intersect.

(i) The purely kinematic case: $\mathcal{V}_{x} \cap F_{x}=\{0\}$ and $T_{x} M=\mathcal{V}_{x}+\left(F_{x} \cap T_{x} M\right)$, for all $x \in M$.

(ii) The case of horizontal symmetries: $\mathcal{V}_{x} \cap F_{x}=\mathcal{V}_{x}$, for all $x \in M$, which is equivalent to $\mathcal{V}_{x} \subset F_{x}$, for all $x \in M$.

(iii) The general case: $\{0\} \mp \mathcal{V}_{x} \cap F_{x} \varsubsetneqq \mathcal{V}_{x}$, for all $x \in M$. 


\section{A. The purely kinematic case}

Suppose that $\mathcal{V}_{x} \cap F_{x}=\{0\}$ and $T_{x} M=\mathcal{V}_{x}+\left(F_{x} \cap T_{x} M\right)$, for all $x \in M$. This implies that $T_{x} M=\mathcal{V}_{x} \oplus\left(F_{x} \cap T_{x} M\right)$. In other words, observing that in this case $U=F \cap T M$, we have $T M$ $=\mathcal{V}_{\mid M} \oplus U$. Since $U$ is $G$-invariant, this decomposition defines a principal connection $\Gamma$ on the principal $G$-bundle $\rho_{\mid M}: M \rightarrow \bar{M}$, with horizontal subspace $U_{x}$ at $x \in M$. Note, in passing, that $U$ here represents a vector bundle of constant rank. In what follows we let $X$ denote a fixed $G$-invariant solution of (15) which, moreover, belongs to $F$. In particular, this means that $X$ is horizontal, i.e., $X \in U$.

Denote by $\mathbf{h}: T M \rightarrow U$ and $\mathbf{v}: T M \rightarrow \mathcal{V}$ the horizontal and vertical projectors associated with the decomposition $T M=\mathcal{V}_{\mid M} \oplus U$. The curvature of $\Gamma$ is the tensor field of type $(1,2)$ on $M$, given by

$$
R=\frac{1}{2}[\mathbf{h}, \mathbf{h}],
$$

where $[$,$] denotes the Nijenhuis bracket of type (1,1)$ tensor fields. Taking into account that in the present case $\bar{U}=T \bar{M}$, and applying the method developed in Sec. V, we obtain on $\bar{M}$ a 2-form $\bar{\omega}$ (which is now a genuine differential form on $\bar{M}$ ) and a function $\bar{H}$ such that the projection $\bar{X}$ of $X$ verifies

$$
i_{\bar{X}} \bar{\omega}=d \bar{H} .
$$

It should be pointed out that the reduced 2-form $\bar{\omega}$ in general need not be closed. We will show, however, that in case the given 2 -form $\omega$ on $P$ is exact, one can construct a reduced equation, equivalent to (20), but now in terms of a closed 2-form on $\bar{M}$.

Assume $\omega=d \theta$ for some 1 -form $\theta$ on $P$. Denote by $\theta^{\prime}$ the 1 -form on $M$ defined by $\theta^{\prime}$ $=j_{M}^{*} \theta$, where $j_{M}: M \hookrightarrow P$ is the canonical inclusion. By means of the given solution $X$ of (15) we can construct a 1-form $\alpha_{X}$ on $M$ as follows:

$$
\alpha_{X}=i_{X}\left(\mathbf{h}^{*} d \theta^{\prime}-d \mathbf{h}^{*} \theta^{\prime}\right),
$$

with the usual convention that, for an arbitrary $p$-form $\beta, \mathbf{h}^{*} \beta$ is the $p$-form defined by the prescription $\mathbf{h}^{*} \beta\left(X_{1}, \ldots, X_{p}\right)=\beta\left(\mathbf{h}\left(X_{1}\right), \ldots, \mathbf{h}\left(X_{p}\right)\right)$.

Lemma VI.1: We have that

$$
\alpha_{X}(Y)=\mathbf{v}(Y)\left(\theta^{\prime}(X)\right)-\theta^{\prime}(R(X, Y))+\theta^{\prime}(\mathbf{h}[X, \mathbf{v}(Y)]),
$$

for all $Y \in \mathfrak{X}(M)$.

Proof: Indeed, for any $Y \in \mathfrak{X}(M)$ we easily find

$$
\begin{aligned}
\alpha_{X}(Y) & =i_{X}\left(\mathbf{h}^{*} d \theta^{\prime}-d \mathbf{h}^{*} \theta^{\prime}\right)(Y) \\
& =\mathbf{h} X\left(\theta^{\prime}(\mathbf{h} Y)\right)-\mathbf{h} Y\left(\theta^{\prime}(\mathbf{h} X)\right)-\theta^{\prime}[\mathbf{h} X, \mathbf{h} Y]-X\left(\theta^{\prime}(\mathbf{h} Y)\right)+Y\left(\theta^{\prime}(\mathbf{h} X)\right)+\theta^{\prime}(\mathbf{h}[X, Y]) \\
& =\mathbf{v} Y\left(\theta^{\prime}(X)\right)-\theta^{\prime}(R(X, Y))+\theta^{\prime}(\mathbf{h}[X, \mathbf{v} Y]),
\end{aligned}
$$

taking into account that $X$ is horizontal.

Q.E.D.

Proposition VI.2: Assume, in addition, that the given action $\Phi$ leaves $\theta$ invariant. Then, the 1-forms $\mathbf{h}^{*} \theta^{\prime}$ and $\alpha_{X}$ are projectable. Moreover, the projection $\bar{X}$ of $X$, which is a solution of (20), also satisfies the equation

$$
i_{\bar{X}} d \bar{\theta}^{\prime}{ }_{h}=d \bar{H}-\overline{\alpha_{X}},
$$

where $\bar{\theta}_{h}^{\prime}$ and $\overline{\alpha_{X}}$ are the projections of the 1-forms $\mathbf{h}^{*} \theta^{\prime}$ and $\alpha_{X}$, respectively.

Proof: We divide the proof in three parts: (i) the $\rho$-projectability of $\mathbf{h}^{*} \theta^{\prime}$; (ii) the $\rho$-projectability of $\alpha_{X}$; (iii) the derivation of the reduced equation of motion (22). 
(i) Let $\xi_{M}$ be the fundamental vector field on $M$ induced by an arbitrary element $\xi \in \mathfrak{g}$. One can readily see that $i_{\xi_{M}} \mathbf{h}^{*} \theta^{\prime}=0$. We now show that also $i_{\xi_{M}} d\left(\mathbf{h}^{*} \theta^{\prime}\right)=0$. Observe that for all $X^{\prime} \in \mathfrak{X}(M)$ we have

$$
i_{\xi_{M}}\left(d \mathbf{h}^{*} \theta^{\prime}\right)\left(X^{\prime}\right)=\xi_{M}\left(\theta^{\prime}\left(\mathbf{h} X^{\prime}\right)\right)+\theta^{\prime}\left(\mathbf{h}\left[\xi_{M}, X^{\prime}\right]\right)
$$

Thus, for $X^{\prime}$ vertical, i.e., $X^{\prime} \in \mathcal{V}$, we obtain $i_{\xi_{M}}\left(d \mathbf{h}^{*} \theta^{\prime}\right)\left(X^{\prime}\right)=0$. Suppose now that $X^{\prime}$ is horizontal, i.e., $X^{\prime} \in U$. Taking into account the $G$-invariance of $\theta^{\prime}$ we deduce that

$$
0=\xi_{M}\left(\theta^{\prime}\left(X^{\prime}\right)\right)-\theta^{\prime}\left(\left[\xi_{M}, X^{\prime}\right]\right)
$$

Herewith we obtain

$$
i_{\xi_{M}}\left(d \mathbf{h}^{*} \theta^{\prime}\right)\left(X^{\prime}\right)=\theta^{\prime}\left(\left[\xi_{M}, X^{\prime}\right]-\mathbf{h}\left[\xi_{M}, X^{\prime}\right]\right)=\theta^{\prime}\left(\mathbf{v}\left[\xi_{M}, X^{\prime}\right]\right)=0
$$

since $X^{\prime}$ is horizontal.

Summarizing, we have shown that each fundamental vector field of the $G$-action on $M$ is a characteristic vector field of $\mathbf{h}^{*} \theta^{\prime}$ and, hence, the latter is a $\rho$-projectable 1 -form.

(ii) To prove the projectability of $\alpha_{X}$ we first note that

$$
i_{\xi_{M}} \alpha_{X}=i_{\xi_{M}} i_{X}\left(\mathbf{h}^{*} d \theta^{\prime}-d \mathbf{h}^{*} \theta^{\prime}\right)=-i_{\xi_{M}} i_{X}\left(d \mathbf{h} * \theta^{\prime}\right)=0
$$

where the last equality follows by a similar argument as above, taking into account that the given $X$ is horizontal.

Next, we prove that $i_{\xi_{M}} d \alpha_{X}=0$. For this it suffices to show that $i_{\xi_{M}} d \alpha_{X}$ vanishes when acting on infinitesimal generators and on horizontal lifts of vector fields on $\bar{M}$. Using the previous property, i.e., $\alpha_{X}\left(\xi_{M}\right)=0$, a straightforward calculation shows that for all $X^{\prime} \in \mathfrak{X}(M)$ :

$$
\left(i_{\xi_{M}} d \alpha_{X}\right)\left(X^{\prime}\right)=\xi_{M}\left(\alpha_{X}\left(X^{\prime}\right)\right)-X^{\prime}\left(\alpha_{X}\left(\xi_{M}\right)\right)-\alpha_{X}\left(\left[\xi_{M}, X^{\prime}\right]\right)=\xi_{M}\left(\alpha_{X}\left(X^{\prime}\right)\right)-\alpha_{X}\left(\left[\xi_{M}, X^{\prime}\right]\right) .
$$

From this we immediately deduce that if $X^{\prime}$ is a fundamental vector field of the group action, $i_{\xi_{M}} d \alpha_{X}\left(X^{\prime}\right)=0$. On the other hand, if $X^{\prime}$ is the horizontal lift of a vector field $Y$ on $\bar{M}$, i.e., $X^{\prime}=Y^{h}$, we obtain, using Lemma VI.1 and the fact that the function $\theta^{\prime}\left(R\left(X, Y^{h}\right)\right)$ is $G$-invariant,

$$
\left(i_{\xi_{M}} d \alpha_{X}\right)\left(Y^{h}\right)=\xi_{M}\left(\alpha_{X}\left(Y^{h}\right)\right)=-\xi_{M}\left(\theta^{\prime}\left(R\left(X, Y^{h}\right)\right)\right)=0
$$

(iii) Recall that $X$ satisfies an equation of the form $i_{X} d \theta=d H+\beta$, for some $\beta \in F^{o}$. Putting $H^{\prime}=j_{M}^{*}\left(=H_{\mid M}\right)$ and $\beta^{\prime}=j_{M}^{*} \beta$, and taking into account that $X$ is tangent to $M$, we can take the pull-back of this equation to $M$ :

$$
i_{X} d \theta^{\prime}=d H^{\prime}+\beta^{\prime} .
$$

Since $X$ is horizontal, i.e., $\mathbf{h} X=X$, it follows that $\mathbf{h}^{*}\left(i_{X} d \theta^{\prime}\right)=i_{X} \mathbf{h}^{*} d \theta^{\prime}$. Furthermore, $H$ (and, hence $H^{\prime}$ ) being $G$-invariant, we have $\mathbf{h}^{*} d H^{\prime}=d H^{\prime}$ and, finally, it is also readily seen that $\mathbf{h}^{*} \beta^{\prime}=0$. The horizontal projection of the equation of motion on $M$ therefore becomes

$$
i_{X} \mathbf{h}^{*} d \theta^{\prime}=d H^{\prime}
$$

In view of the definition of the 1 -form $\alpha_{X}$, we then obtain

$$
i_{X} d \mathbf{h}^{*} \theta^{\prime}=i_{X} \mathbf{h}^{*} d \theta^{\prime}-\alpha_{X}=d H^{\prime}-\alpha_{X}
$$

All terms in this equation are projectable onto $\bar{M}$ and the reduced equation is indeed given by (22). 
Proposition 6.2 describes a situation where a constrained Hamiltonian system (15) with symmetry, admits a reduction to an unconstrained system (22), but with an additional "nonconservative force" represented by $\overline{\alpha_{X}}$. It is interesting to observe that, by construction, the 1 -form $\alpha_{X}$ satisfies

$$
i_{X} \alpha_{X}=0
$$

We now briefly comment on the problem of reconstructing the dynamics on $M$ from the reduced dynamics on $\bar{M}$ in the case where (15) admits a unique solution $X$. Suppose the flow of the reduced system $\bar{X}$ is known. In order to recover flow of the constrained dynamics on $M$, one can first lift the integral curves of $\bar{X}$ to $M$ by means of the horizontal lift operation associated with the principal connection $\Gamma$. The integral curves of $X$ are then obtained by "shifting" these lifted curves along the fibres of $\rho_{\mid M}$. This second step can be implemented in the standard way. ${ }^{37,38}$

Finally, we can summarize the situation in the case of purely kinematic constraints in the following diagram:

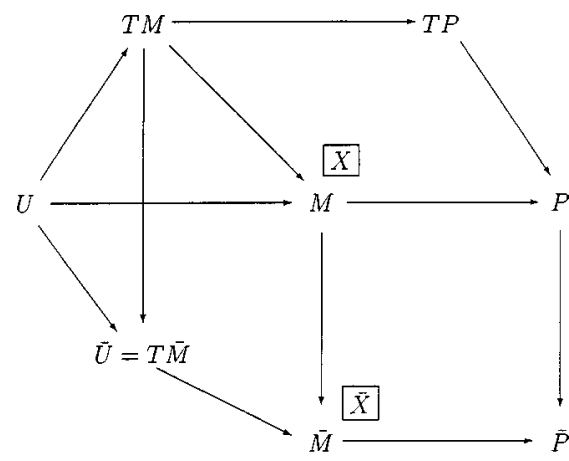

\section{B. The case of horizontal symmetries}

The assumption now is that $\mathcal{V}_{x} \cap F_{x}=\mathcal{V}_{x}$, for all $x \in M$ or, equivalently, $\mathcal{V}_{\mid M} \subset F$. In particular, every infinitesimal generator of the given group action then yields a horizontal symmetry as defined at the beginning of this section. Note also that an unconstrained Hamiltonian system with symmetry can be regarded as a special subcase of this case, since we then have $M=P, F=T P$ and, obviously, $\mathcal{V} \subset T P$.

For the further analysis of this case we assume, in addition, that the given symplectic action $\Phi$ on $P$ is a Hamiltonian action, in the sense that it admits an $\mathrm{Ad}^{*}$-equivariant momentum map $J: P \rightarrow \mathfrak{g}^{*}$, such that for all $\xi \in \mathfrak{g}, i_{\xi_{P}} \omega=d\langle J, \xi\rangle$. Let $\mu \in \mathfrak{g}^{*}$ be a regular value of $J$, and suppose that the isotropy group $G_{\mu}$ acts freely and properly on the level set $J^{-1}(\mu)$. It is known (see Refs. 37, 36) that under these conditions $\left(P_{\mu}=J^{-1}(\mu) / G_{\mu}, \omega_{\mu}\right)$ is a symplectic manifold, where $\omega_{\mu}$ is the 2 -form defined by

$$
\pi_{\mu}^{*} \omega_{\mu}=j_{\mu}^{*} \omega
$$

with $\pi_{\mu}: J^{-1}(\mu) \rightarrow P_{\mu}$ the canonical projection and $j_{\mu}: J^{-1}(\mu) \hookrightarrow P$ the natural inclusion.

With $\xi_{P}$ again denoting the infinitesimal generator of the group action on $P$, corresponding to an element $\xi \in \mathfrak{g}$, it follows from the definition of the momentum mapping that $\xi_{P}=X_{J_{\xi}}$, where $J_{\xi}(x)=J(x)(\xi)$ for all $x \in P$. Taking into account that, by assumption, $\mathcal{V}_{\mid M} \subset F$, we find that for any solution $X$ of (15), along the constraint submanifold $M$,

$$
X\left(J_{\xi}\right)=0,
$$


i.e., the components of the momentum mapping are conserved quantities for the constrained dynamics. This is a version of Noether's theorem for constrained systems. (For the case of mechanical systems with nonholonomic constraints, see in this respect also Refs. 15, 39, 35.)

Imposing a condition of clean intersection of $M$ and $J^{-1}(\mu)$, we have that $M^{\prime}$ $=M \cap J^{-1}(\mu)$ is a submanifold of $J^{-1}(\mu)$ which is $G_{\mu}$-invariant. Passing to the quotient we then obtain a submanifold $M_{\mu}=M^{\prime} / G_{\mu}$ of $P_{\mu}$. Next, we can define a distribution $F^{\prime}$ on $P$ along $M^{\prime}$ by putting

$$
F_{x^{\prime}}^{\prime}=T_{x^{\prime}}\left(J^{-1}(\mu)\right) \cap F_{x^{\prime}}, \quad \forall x^{\prime} \in M^{\prime},
$$

and we now make the further simplifying assumption that $F^{\prime}$ has constant rank. It is obvious that $F^{\prime}$ is a $G_{\mu}$-invariant subbundle of $T P_{\mid M^{\prime}}$ and, hence, it projects onto a subbundle $F_{\mu}$ of $T P_{\mu}$ along $M_{\mu}$. Finally, since the restriction of the Hamiltonian $H$ to $J^{-1}(\mu)$ is also $G_{\mu}$-invariant, it induces a function $H_{\mu}$ on $P_{\mu}$.

Theorem VI.3: Suppose that $X$ is a $G$-invariant solution of (15). Then, $X$ induces a vector field $X_{\mu}$ on $M_{\mu}$, such that

$$
\left(i_{X_{\mu}} \omega_{\mu}-d H_{\mu}\right)_{\mid M_{\mu}} \in F_{\mu}^{o}, \quad X_{\mu} \in T M_{\mu} .
$$

Proof: First of all, notice that $X^{\prime}=X_{\mid M^{\prime}}$ is everywhere tangent to $M^{\prime}$, since both $J^{-1}(\mu)$ and $M$ are invariant submanifolds of $X$. Pulling back (15) to $J^{-1}(\mu)$, we find that $X^{\prime}$ satisfies an equation of the form

$$
\left(i_{X^{\prime}} j_{\mu}^{*} \omega-d\left(H \circ j_{\mu}\right)\right)_{\mid M^{\prime}}=\beta
$$

for some section $\beta$ of $F^{\prime o}$. Since $X$ is $G$-invariant, and taking account the other symmetry assumptions, it follows that both $X^{\prime}$ and $\beta$ are $G_{\mu}$-equivariant sections of $T M^{\prime}$ and $F^{\prime o}$, respectively. Moreover, from the fact that we are dealing with horizontal symmetries we may deduce, in particular, that for all $\xi \in \mathfrak{g}_{\mu}$ (= the Lie algebra of $\left.G_{\mu}\right),\left(\xi_{P}\right)_{\mid M^{\prime}}$ is a section of $F^{\prime}$. Therefore, $\beta$ projects onto a section of $F_{\mu}^{o}$. Using a standard argument, it now readily follows that $X^{\prime}$ projects onto a vector field on $M_{\mu}$ for which (23) holds.

Q.E.D.

In the case of horizontal symmetries we have thus proved that, under the appropriate assumptions, the given constrained problem on $(P, \omega)$ reduces to a constrained problem on $\left(P_{\mu}, \omega_{\mu}\right)$.

As far as the reconstruction of the original constrained dynamics from the reduced dynamics is concerned, we observe that, unlike in the purely kinematic case, we now first have to select an arbitrary connection on the principal $G_{\mu}$-bundle $M^{\prime} \rightarrow M_{\mu}$. This connection will enable us to subsequently lift the integral curves of the reduced system from $M_{\mu}$ to $M^{\prime}$. The reconstruction of the flow of $X$ then further proceeds as in the previous case.

The following diagram illustrates the situation in the case of horizontal symmetries. Note in passing that, modulo the appropriate embeddings, one may identify $M_{\mu}$ with $\bar{M} \cap P_{\mu}$ where, as before, $\bar{M}=M / G$.

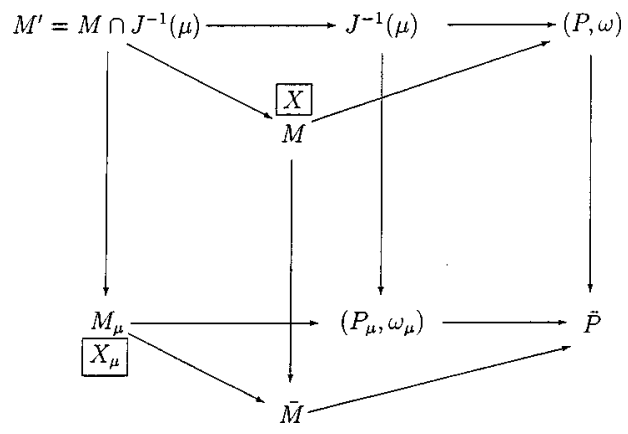




\section{The general case}

We now consider the case where, at $x \in M,\{0\} \neq \mathcal{V}_{x} \cap F_{x} \neq \mathcal{V}_{x}$. Assuming again that the given action of $G$ on $P$ is Hamiltonian, with momentum map $J$, it is no longer true that $J$ is a conserved quantity for the constrained dynamics. However, extending a procedure developed by Bloch et al. ${ }^{15}$ for nonholonomic mechanical systems (see also Ref. 40), we will derive an equation which describes the evolution of some components of the momentum map along the integral curves of the constrained system.

For each $x \in M$, we put

$$
\mathfrak{g}^{x}=\left\{\xi \in \mathfrak{g} \mid \xi_{M}(x) \in F_{x}\right\},
$$

and

$$
S^{x}=\left\{\xi_{M}(x) \mid \xi \in \mathfrak{g}^{x}\right\},
$$

i.e., $S^{x}=\mathcal{V}_{x} \cap F_{x}$. Recall that $\xi_{M}$ is just the restriction of $\xi_{P}$ to the $G$-invariant submanifold $M$. We have that $\mathfrak{g}^{x}$ and $S^{x}$ are vector subspaces of $\mathfrak{g}$ and $T_{x} M\left(\subset T_{x} P\right)$, respectively. Putting

$$
\mathfrak{g}^{F}=\underset{x \in M}{\amalg} \mathfrak{g}^{x}, \quad S^{F}=\underset{x \in M}{\amalg} S^{x},
$$

where we use the symbol " $U$ " to denote the disjoint union of the respective vector spaces, we obtain two ("generalized") vector bundles over $M$, with corresponding natural projections $\mathfrak{g}^{F}$ $\rightarrow M: \xi \in \mathfrak{g}^{x} \mapsto x$ and $S^{F} \rightarrow M: \xi_{M}(x) \mapsto x$. In general, these bundles need not have constant rank. However, for the subsequent discussion we make the simplifying assumption that $\mathfrak{g}^{F}$ and $S^{F}$ are genuine vector bundles over $M$, the fibers of which have constant dimension (independent of the base point). The given action being a free action, the mapping $\mathfrak{g}^{F} \rightarrow S^{F}: \xi \in \mathfrak{g}^{x} \mapsto \xi_{M}(x)$ then defines a smooth vector bundle isomorphism.

Suppose now that the symplectic form $\omega$ is exact, say $\omega=d \theta$, and that the $G$-action leaves $\theta$ invariant. In such a case there always exists a well-defined momentum mapping $J: P \rightarrow \mathfrak{g}^{*}$ such that

$$
\langle J(x), \xi\rangle=-\left(\theta_{x}\right)\left(\xi_{P}(x)\right), \quad \forall x \in P, \quad \forall \xi \in \mathfrak{g}
$$

(see, e.g., Ref. 37). Herewith we can define a smooth section $J^{(c)}: M \rightarrow\left(\mathfrak{g}^{F}\right)^{*}$ of the dual bundle $\left(\mathfrak{g}^{F}\right)^{*}$ as follows:

$$
J^{(c)}(x): \mathfrak{g}^{x} \rightarrow \mathbb{R}, \quad J^{(c)}(x)(\xi)=\langle J(x), \xi\rangle .
$$

We may call $J^{(c)}$ the "constrained momentum map." In Ref. 15, which deals with nonholonomic mechanical systems, this map was denoted by $J^{\text {nhc }}$. Given a smooth section $\bar{\xi}$ of the vector bundle $\mathfrak{g}^{F}$, we can then define a smooth function $J_{\bar{\xi}}^{(c)}$ on $M$ according to

$$
J_{\bar{\xi}}^{(c)}=\left\langle J^{(c)}, \bar{\xi}\right\rangle .
$$

In addition, we can construct a vector field $\Xi$ on $M$ by putting

$$
\Xi(x)=(\bar{\xi}(x))_{M}(x), \quad \forall x \in M .
$$

Denoting the Lie derivative operator with respect to $\Xi$ as $\mathcal{L}_{\Xi}$, we have the following interesting result.

Theorem VI.4: Let $X$ be an arbitrary solution of (15). For any smooth section $\bar{\xi}$ of $\mathfrak{g}^{F}$ we then have 


$$
X\left(J_{\bar{\xi}}^{(c)}\right)=-\left(\mathcal{L}_{\Xi} \theta\right)(X)
$$

Proof: Since $\Xi$ takes values in $F$, it follows from (15) that, along $M$,

$$
i_{\Xi} i_{X} \omega-i_{\Xi} d H=0 .
$$

From the above definitions we further deduce that $J_{\bar{\xi}}^{(c)}=-i_{\Xi}\left(j_{M}^{*} \theta\right)$, with $j_{M}: M \hookrightarrow P$ again denoting the inclusion map. A straightforward computation then gives

$$
X\left(J_{\bar{\xi}}^{(c)}\right)=-i_{X} d i_{\Xi}\left(j_{M}^{*} \theta\right)=-i_{X} \mathcal{L}_{\Xi}\left(j_{M}^{*} \theta\right)+i_{X} i_{\Xi}\left(j_{M}^{*} \omega\right)=-\mathcal{L}_{\Xi} i_{X}\left(j_{M}^{*} \theta\right)+i_{[\Xi, X]}\left(j_{M}^{*} \theta\right)-\Xi\left(H \circ j_{M}\right) .
$$

Since $H$ is $G$-invariant, it follows from the definition of $\Xi$ that $\Xi\left(H \circ j_{M}\right)=0$. Herewith, the previous relation immediately reduces to (24) (with a slight abuse of notation). Q.E.D.

Note that for the above result we do not have to require $X$ to be $G$-invariant. Equation (24) is called the momentum equation for the given constrained system. In the case of linear nonholonomic constraints we precisely recover the result established by Bloch et al. ${ }^{15}$

Suppose again that $X$ is a solution of (15) and let $\bar{\xi}$ be a constant section of $\mathfrak{g}^{F}$, i.e., $\bar{\xi}(x)$ $=\xi^{0} \in \mathfrak{g}$ for all $x \in M$. We may then identify the corresponding vector field $\Xi$ with the infinitesimal generator $\xi_{M}^{0}$ and, clearly, $J_{\bar{\xi}}^{(c)}=\left(J_{\xi^{0}}\right)_{\mid M}$. Moreover, by construction, $\xi_{M}^{0}$ is a horizontal symmetry. The momentum equation (24) then leads to

$$
X\left(J_{\bar{\xi}}^{(c)}\right)=X\left(J_{\xi^{0}}\right)_{\mid M}=0
$$

i.e., we have obtained a conserved quantity of $X$ associated with the horizontal symmetry $\xi_{M}^{0}$. This is again a manifestation of Noether's theorem for constrained systems (cf. the previous subsection).

In the next section we will apply some of the previous results to the case of a singular Lagrangian system and to a Lagrangian system with linear nonholonomic constraints induced by a principal connection.

\section{APPLICATIONS}

\section{A. Singular Lagrangian systems}

Consider a system described by a singular Lagrangian function $L: T Q \rightarrow \mathbb{R}$ such that $\omega_{L}$ is presymplectic. We assume that a Lie group $G$ acts freely and properly on the configuration manifold $Q$ and that $L$ is invariant under the lifted action of $G$ on $T Q$. It then easily follows that both $\omega_{L}$ and $E_{L}$ are also $G$-invariant. In addition, we know that the lifted action of $G$ on $T^{*} Q$ leaves invariant the Liouville 1-form $\theta_{Q}$ and, hence, also the canonical symplectic form $\omega_{Q}=$ $-d \theta_{Q}$ (see, e.g., Ref. 37). From all this, one can subsequently deduce that the Legendre mapping is $G$-equivariant and that the constraint submanifolds generated by the presymplectic constraint algorithm, both on the Lagrangian and on the Hamiltonian side, are $G$-invariant. In particular, the final constraint submanifold $M_{f}$ in $T^{*} Q$ is $G$-invariant.

Let us now consider the constrained equations of motion (7) where, for simplicity, we write $H$ instead of $H_{1}$, i.e.,

$$
\left(i_{X} \omega_{Q}-d H\right)_{\mid M_{f}} \in T M_{1}^{o}, \quad X_{\mid M_{f}} \in T M_{f} .
$$

Since $M_{f}$ is $G$-invariant, it follows that $\mathcal{V}_{\mid M_{f}} \subset T M_{f} \subseteq T M_{1}=F$ and, hence, we are in the case of horizontal symmetries. Moreover, the lifted symplectic action of $G$ on $T^{*} Q$ admits an equivariant momentum map $J$ and so we can apply the reduction procedure described in subsection IV B. Given a regular value $\mu$ of $J$, it is easy to check that the reduced system then becomes

$$
\left(i_{X_{\mu}} \omega_{\mu}-d H_{\mu}\right)_{\mid\left(M_{f}\right)_{\mu}} \in\left(T\left(M_{1}\right)_{\mu}\right)^{o}, \quad X_{\mu} \in T\left(M_{f}\right)_{\mu},
$$


whereby we observe that

$$
\left(T\left(M_{1}\right)_{\mu}\right)^{o} \subset\left(T\left(M_{f}\right)_{\mu}\right)^{o}
$$

Suppose, on the other hand, we would have started from the description of the given constrained system in terms of (8), again denoting the extended Hamiltonian by $H$, i.e.,

$$
\left(i_{X} \omega_{Q}-d H\right)_{\mid M_{f}} \in T M_{f}^{o}, \quad X_{\mid M_{f}} \in T M_{f}
$$

Under the given assumptions, the final constraint submanifold $M_{f}$, generated through Hinds' algorithm, will also be $G$-invariant such that $\mathcal{V}_{\mid M_{f}} \subset F=T M_{f}$, i.e., we are again in the case of horizontal symmetries. Given a regular value $\mu$ of the momentum map $J$, it is easy to check that we now have

$$
\left(T M_{f}\right)_{\mu}^{o}=\left(T\left(M_{f}\right)_{\mu}\right)^{o}
$$

where, assuming clean intersection of $M_{f}$ and $J^{-1}(\mu), \quad\left(M_{f}\right)_{\mu}=\left(M_{f} \cap J^{-1}(\mu)\right) / G_{\mu}$ and $\left(T M_{f}\right)_{\mu}=\left(T J^{-1}(\mu) \cap T M_{f}\right) / G_{\mu}$. If (25) admits a $G$-invariant solution $X$, it follows from Theorem 6.3 that the reduced dynamics will satisfy the constrained system

$$
\left(i_{X_{\mu}} \omega_{\mu}-d H_{\mu}\right)_{\mid\left(M_{f}\right)_{\mu}} \in\left(T\left(M_{f}\right)_{\mu}\right)^{o}, \quad X_{\mu} \in T\left(M_{f}\right)_{\mu} .
$$

We now have the following diagram:

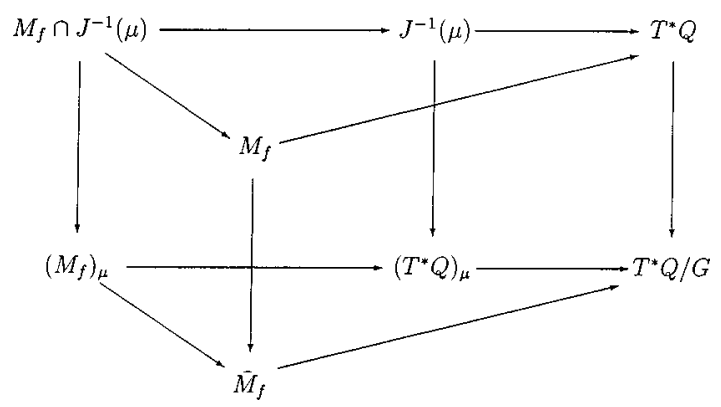

Notice that, according to Proposition 3.1, the reduced system (26) admits a unique solution if and only if $T\left(M_{f}\right)_{\mu}^{\perp} \cap T\left(M_{f}\right)_{\mu}=0$, which implies that $\left(M_{f}\right)_{\mu}$ is a symplectic submanifold of $\left(T^{*} Q\right)_{\mu}$. In that case we have the direct sum decomposition

$$
T\left(\left(T^{*} Q\right)_{\mu}\right)_{\mid\left(M_{f}\right)_{\mu}}=T\left(M_{f}\right)_{\mu} \oplus T\left(M_{f}\right)_{\mu}^{\perp}
$$

and we can construct the unique solution of (26) in the following way. Let $X_{H_{\mu}}$ denote the Hamiltonian vector field on $\left(\left(T^{*} Q\right)_{\mu}, \omega_{\mu}\right)$, corresponding to $H_{\mu}$. The reduction $X_{\mu}$ of $X$ is then obtained by first taking the restriction of $X_{H_{\mu}}$ to $\left(M_{f}\right)_{\mu}$, and then projecting it onto $T\left(M_{f}\right)_{\mu}$.

Example: Consider the singular Lagrangian function $L: T \mathbb{R}^{6} \rightarrow \mathbb{R}$ given by

$$
L=m_{2}\left(\dot{x}_{2}^{2}+\dot{y}_{2}^{2}\right)+m_{3}\left(\dot{x}_{3}^{2}+\dot{y}_{3}^{2}\right)+\dot{y}_{2} x_{2}-\dot{x}_{2} y_{2}+\dot{y}_{3} x_{3}-\dot{x}_{3} y_{3}-x_{1}^{2}-y_{1}^{2}-x_{2}^{2}-y_{2}^{2}-x_{3}^{2}-y_{3}^{2},
$$

with coordinates $\left(x_{1}, y_{1}, x_{2}, y_{2}, x_{3}, y_{3}, \dot{x}_{1}, \dot{y}_{1}, \dot{x}_{2}, \dot{y}_{2}, \dot{x}_{3}, \dot{y}_{3}\right)$ on $T R^{6}$. Here $m_{2}$ and $m_{3}$ are constants. The above Lagrangian is a particular case of those considered by Capri and Kobayashi ${ }^{41,42}$ (see also Ref. 43). This type of Lagrangian occurs in some models of field theories coupled to external fields.

When passing to the Hamiltonian side, we obtain the following two primary constraints: $\phi_{1}$ $=p_{x_{1}}=0$ and $\phi_{2}=p_{y_{1}}=0$ which determine the constraint submanifold $M_{1}$. The 2-form $\omega_{1}$ is given in local coordinates $\left(x^{1}, y^{1}, x^{2}, y^{2}, x^{3}, y^{3}, p_{x_{2}}, p_{y_{2}}, p_{x_{3}}, p_{y_{3}}\right)$ on $M_{1}$ by 


$$
\omega_{1}=d x_{2} \wedge d p_{x^{2}}+d y_{2} \wedge d p_{y^{2}}+d x_{3} \wedge d p_{x^{3}}+d y_{3} \wedge d p_{y^{3}},
$$

which is presymplectic, with

$$
\operatorname{ker} \omega_{1}=\left\langle\frac{\partial}{\partial x_{1}}, \frac{\partial}{\partial y_{1}}\right\rangle
$$

The energy function $E_{L}$ projects onto the function $h_{1}$ on $M_{1}$ given by

$$
\begin{aligned}
h_{1}= & \frac{1}{4 m_{2}}\left(\left(p_{x_{2}}+y_{2}\right)^{2}+\left(p_{y_{2}}-x_{2}\right)^{2}\right)+\frac{1}{4 m_{3}}\left(\left(p_{x_{3}}+y_{3}\right)^{2}+\left(p_{y_{3}}-x_{3}\right)^{2}\right) \\
& +\left(x_{1}\right)^{2}+\left(y_{1}\right)^{2}+\left(x_{2}\right)^{2}+\left(y_{2}\right)^{2}+\left(x_{3}\right)^{2}+\left(y_{3}\right)^{2} .
\end{aligned}
$$

Consistency of the constraints $\phi_{1}$ and $\phi_{2}$ leads to the secondary constraints

$$
\phi_{3}=x_{1}=0, \quad \phi_{4}=y_{1}=0,
$$

and the constraint submanifold $M_{2}$ determined by the vanishing of the constraints $\phi_{i}, 1 \leqslant i \leqslant 4$, turns out to be the final constraint submanifold, i.e., $M_{2}=M_{f}$. We note, in passing, that in this case the final constraint submanifolds generated by the Gotay-Nester algorithm and the Hinds algorithm, coincide.

Consider the function $H$ on $T^{*} Q$ with coordinate expression equal to that of $h_{1}$. (In fact, one might take any arbitrary extension of $h_{1}$ which coincides with the latter on $M_{f}$; for instance: $\widetilde{H}$ $=H+\lambda_{1} \phi_{1}+\lambda_{2} \phi_{2}$ for some arbitrary functions $\lambda_{i}$ ). Note also that

$$
T M_{f}^{\perp}=\left\langle\frac{\partial}{\partial x_{1}}, \frac{\partial}{\partial y_{1}}, \frac{\partial}{\partial p_{x_{1}}}, \frac{\partial}{\partial p_{y_{1}}}\right\rangle .
$$

The constrained equations of motion (8), i.e.,

$$
i_{X} \omega_{Q}-d H \in T M_{f}^{o}, \quad X \in T M_{f},
$$

admit a unique solution since $T M_{f} \cap T M_{f}^{\perp}=0$.

The initial system admits nongauge symmetries which are rotations on the configuration space. The action

$$
\Phi: T^{2} \times R^{6} \rightarrow R^{6},
$$

where $\mathbb{T}^{2}$ is the two-dimensional torus, is given by

$$
\begin{aligned}
\Phi\left(\left(\theta_{2}, \theta_{3}\right) \times\left(x^{1}, y^{1}, x^{2}, y^{2}, x^{3}, y^{3}\right)\right)= & \left(x_{1}, y_{1}, x_{2} \sin \theta_{2}+y_{2} \cos \theta_{2}, x_{2} \cos \theta_{2}\right. \\
& \left.-y_{2} \sin \theta_{2}, x_{3} \sin \theta_{3}+y_{3} \cos \theta_{3}, x_{3} \cos \theta_{3}-y_{3} \sin \theta_{3}\right) .
\end{aligned}
$$

The infinitesimal generators of this action are

$$
\left\langle x_{i} \frac{\partial}{\partial y_{i}}-y_{i} \frac{\partial}{\partial x_{i}}\right\rangle, \quad 2 \leqslant i \leqslant 3 .
$$

The infinitesimal generators of the lifted action to $T^{*} Q$ are

$$
\left\langle x_{i} \frac{\partial}{\partial y_{i}}-y_{i} \frac{\partial}{\partial x_{i}}+p_{x_{i}} \frac{\partial}{\partial p_{y_{i}}}-p_{y_{i}} \frac{\partial}{\partial p_{x_{i}}}\right\rangle, \quad 2 \leqslant i \leqslant 3 .
$$


This lifted action is symplectic and preserves the Hamiltonian $H$. Moreover, the manifold $M_{f}$ is also $\mathbb{T}^{2}$-invariant.

According to our classification of constrained systems, we are in the case of horizontal symmetries, since at each point $x \in M_{f}$,

$$
\mathcal{V}_{x} \cap\left(F_{x} \cap T_{x} M_{f}\right)=\mathcal{V}_{x},
$$

because $F=T M_{f}$.

Consider the equivariant momentum map $J: T^{*} Q \rightarrow R^{2}$ given by

$$
J\left(x_{1}, y_{1}, x_{2}, y_{2}, x_{3}, y_{3} ; p_{x_{1}}, p_{y_{1}}, p_{x_{2}}, p_{y_{2}}, p_{x_{3}}, p_{y_{3}}\right)=\left(x_{2} p_{y_{2}}-y_{2} p_{x_{2}}, x_{3} p_{y_{3}}-y_{3} p_{x_{3}}\right) \text {. }
$$

For any regular value $\mu=\left(\mu_{1}, \mu_{2}\right)$, applying the cotangent bundle reduction, we have that $J^{-1}(\mu) /\left(\mathbb{T}^{2}\right)_{\mu}$ is a differentiable manifold equipped with a symplectic 2-form $\omega_{\mu}$. Denote by $H_{\mu}$ the projection of $H_{\mid J^{-1}(\mu)}$, and by $\left(M_{f}\right)_{\mu}$ the projection of $M_{f} \cap J^{-1}(\mu)$, with projection map $\pi_{\mu}: J^{-1} \mu \rightarrow J^{-1}(\mu) /\left(\mathbb{T}^{2}\right)_{\mu}$. Then, from Theorem 6.3 it follows that the solution of system (27) projects onto the solution of the system

$$
\left(i_{X_{\mu}} \omega_{\mu}-d H_{\mu}\right)_{\mid\left(M_{f}\right)_{\mu}} \in\left(T\left(M_{f}\right)_{\mu}\right)^{o}, \quad X_{\mu} \in T\left(M_{f}\right)_{\mu} .
$$

By taking polar coordinates on $M_{f}$, i.e., $\left(r_{2}, \varphi_{2}, r_{3}, \varphi_{3} ; p_{r_{2}}, p_{\varphi_{2}}, p_{r_{3}}, p_{\varphi_{3}}\right)$, we have that $J^{-1}(\mu) \cap M_{f}$ is the $\left(\mathbb{T}^{2}\right)_{\mu}$-invariant submanifold of $M_{f}$ determined by

$$
p_{\varphi_{2}}=\mu_{1} \quad \text { and } \quad p_{\varphi_{3}}=\mu_{2} .
$$

Passing to the quotient we find that $\left(M_{f}\right)_{\mu}$ is a four-dimensional submanifold of $J^{-1}(\mu) /\left(\mathbb{T}^{2}\right)_{\mu}$, with induced coordinates $\left(r_{2}, r_{3} ; p_{r_{2}}, p_{r_{3}}\right)$ and equipped with the symplectic form

$$
\left(\omega_{M_{f}}\right)_{\mu}=d r_{2} \wedge d p_{r_{2}}+d r_{3} \wedge d p_{r_{3}} .
$$

\section{B. Nonholonomic Lagrangian systems}

We again consider an action of a Lie group $G$ on a manifold $Q$, and let $L: T Q \rightarrow \mathrm{R}$ be a regular Lagrangian which is $G$-invariant. The lifted action of $G$ on the symplectic manifold $\left(T Q, \omega_{L}\right)$ is then Hamiltonian. We assume that the Lagrangian system is subjected to some linear nonholonomic constraints, described by a distribution $\mathcal{D}$ on $Q$, such that the resulting nonholonomic system verifies the compatibility condition (cf. Sec. II B) and such that, in addition, the vector subbundle $D$ of $T Q$, spanned by $\mathcal{D}$, is $G$-invariant. The constrained equations then read (cf. (9))

$$
i_{X} \omega_{L}-d E_{L} \in\left(\mathcal{D}^{v}\right)^{o}, \quad X_{\mid D} \in T D .
$$

We now consider an interesting special subcase of the purely kinematic case, namely, a (generalized) Caplygin system. For a system of Caplygin type, the configuration manifold $Q$ is a principal $G$-bundle $\pi: Q \rightarrow Q / G$, and the constraints are given by the horizontal subspaces of a principal connection $\Gamma$ on $\pi$ (see Refs. 12, 24).

Under the above conditions, one can easily see that there exists a well-defined Lagrangian function $L^{*}: T(Q / G) \rightarrow \mathrm{R}$, given by

$$
L^{*}(Y)=L\left(\left(Y^{h}\right)_{q}\right),
$$

for any $Y \in T_{y}(Q / G)$, where $q \in Q$ is an arbitrary point in the fiber over $y \in Q / G$ and $Y^{h}$ denotes the horizontal lift of $Y$ with respect to $\Gamma$.

A direct computation shows that, with the notations introduced in $\mathrm{Sec} . \mathrm{V}, \mathcal{V} \cap \mathcal{D}^{v}=0$. Moreover, we have $U=\mathcal{D}^{v} \cap T D$, and $U$ is symplectic with respect to $\omega_{L}$. Therefore we deduce that 


$$
T D=\mathcal{V} \oplus U
$$

Thus, a Caplygin system fits indeed very nice in the purely kinematic case. Moreover, one can prove that $\bar{D}=D / G \cong T(Q / G)$ and $\bar{E}_{L}=E_{L^{*}}$.

We have seen that the compatibility condition,

$$
\left(\mathcal{D}^{v}\right)^{\perp} \cap T D=0,
$$

ensures the existence of a unique solution $X=\Gamma_{L, D}$ of (28) which, moreover, is a SODE. Notice that $\Gamma_{L, D}$ can be obtained by projecting the unconstrained Euler-Lagrange vector field $\Gamma_{L}$ by means of the first projector associated with the decomposition,

$$
T(T Q)_{\mid D}=T D \oplus\left(\mathcal{D}^{v}\right)^{\perp}
$$

Since $\omega_{L}=-d \theta_{L}$, the reduced equation becomes

$$
i_{X}^{-} \omega_{L^{*}}=d E_{L^{*}}-\overline{\alpha_{\Gamma_{L, D}}}
$$

where $\overline{\alpha_{\Gamma_{L, D}}}$ is the projection of the 1-form $\alpha_{\Gamma_{L, D}}$, defined by (21). Observe that

$$
i_{\Gamma} \overline{\alpha_{\Gamma_{L, D}}}=0
$$

for any $\operatorname{SODE} \Gamma$ on $T(Q / G)$. This implies that $\overline{\alpha_{\Gamma_{L, D}}}$ is a 1 -form of gyroscopic type.

Example VII.1: The vertical rolling disk. Consider a rolling disk of radius $R$ constrained to remain vertical on a horizontal plane. The standard coordinates of the configuration space $\mathbb{R}$ $\times S^{1} \times S^{1}$ are: the Cartesian coordinates $x, y$ of the center of mass, the angle $\theta_{1}$ between the tangent of the disk at the point of contact and the $x$-axis and the angle $\theta_{2}$ determined by some diameter of the disk and the vertical.

The dynamics of this mechanical system is described by the following:

$$
L=\frac{1}{2}\left(m \dot{x}^{2}+m \dot{y}^{2}+I_{1} \dot{\theta}_{1}^{2}+I_{2} \dot{\theta}_{2}^{2}\right),
$$

where $m$ is the mass, and $I_{1}, I_{2}$ are moments of inertia;

the nonholonomic constraints:

$$
\phi_{1}=\dot{x}-\left(R \cos \theta_{1}\right) \dot{\theta}_{2}=0, \quad \phi_{2}=\dot{y}-\left(R \sin \theta_{1}\right) \dot{\theta}_{2}=0 .
$$

The Poincaré-Cartan 2-form of the Lagrangian $L$ is

$$
\omega_{L}=m d x \wedge d \dot{x}+m d y \wedge d \dot{y}+I_{1} d \theta_{1} \wedge d \dot{\theta}_{1}+I_{2} d \theta_{2} \wedge d \dot{\theta}_{2},
$$

so that the Euler-Lagrange vector field of the free (i.e., unconstrained system) is

$$
\Gamma_{L}=\dot{x} \frac{\partial}{\partial x}+\dot{y} \frac{\partial}{\partial y}+\dot{\theta}_{1} \frac{\partial}{\partial \theta_{1}}+\dot{\theta}_{2} \frac{\partial}{\partial \theta_{2}} .
$$

Consider the group $G=\mathbb{R}^{2}$ and its trivial action by translations on $Q$ :

$$
\begin{gathered}
\Phi: G \times Q \rightarrow Q \\
(r, s) \times\left(x, y, \theta_{1}, \theta_{2}\right) \mapsto\left(x+r, y+s, \theta_{1}, \theta_{2}\right) .
\end{gathered}
$$

If we consider the lifted action $\Phi^{1}$ of $\Phi$ to $T Q$, given by $\left(\Phi^{1}\right)_{g}=T \Phi_{g}$, then the infinitesimal generators of this action are 


$$
\mathcal{V}=\left\langle\frac{\partial}{\partial x}, \frac{\partial}{\partial y}\right\rangle
$$

One easily verifies that the constraint submanifold $D$, determined by $\phi_{1}, \phi_{2}$, is invariant with respect to $\Phi^{1}$. Choose local coordinates $\left(x, y, \theta_{1}, \theta_{2}, \dot{\theta}_{1}, \dot{\theta}_{2}\right)$ on $D$. In these coordinates we find that the distribution $U$ on $D$ is generated by the vector fields:

$$
U=\left\langle\frac{\partial}{\partial \theta_{1}}, \frac{\partial}{\partial \theta_{2}}, R \cos \theta_{1} \frac{\partial}{\partial x}+R \sin \theta_{1} \frac{\partial}{\partial y}+\frac{\partial}{\partial \dot{\theta}_{2}}, \frac{\partial}{\partial \dot{\theta}_{1}}\right\rangle,
$$

and we readily have that $\mathcal{V}_{\mid D} \subset T D$ and $\mathcal{V}_{\mid D} \oplus U=T D$, i.e., we are in the purely kinematic case. In fact, noting that $\rho: Q \rightarrow S^{1} \times S^{1}$ is a principal bundle, with structure group $G=\mathbb{R}^{2}$, and $D$ is the horizontal subbundle of a principal connection, we see that the given system is a Caplygin system. Following the above analysis we then obtain

$$
\begin{gathered}
L^{*}=\frac{1}{2}\left(I_{1} \dot{\theta}_{1}^{2}+\left(m R^{2}+I_{2}\right) \dot{\theta}_{2}^{2}\right), \\
\omega_{L^{*}}=I_{1} d \theta_{1} \wedge d \dot{\theta}_{1}+\left(m R^{2}+I_{2}\right) d \theta_{2} \wedge d \dot{\theta}_{2} .
\end{gathered}
$$

In this particular case the gyroscopic 1-form $\overline{\alpha_{\Gamma_{L, D}}}=0$ and then the 2 -form $\omega_{U}^{-}$is closed and

$$
\omega_{\bar{U}}^{-}=\omega_{L *}
$$

Example VII.2: The two-wheeled carriage. The configuration space of the two-wheeled carriage is $Q=\mathrm{R}^{2} \times S^{1} \times \mathrm{T}^{2}$ with coordinates $\left(x, y, \varphi, \Psi_{1}, \Psi_{2}\right)$ (see, e.g., Ref. 24 for more details).

This system is determined by the following data:

(i) A regular Lagrangian $L$,

$$
L=\frac{1}{2} m\left(\dot{x}^{2}+\dot{y}^{2}\right)+m_{0} l \dot{\varphi}(\dot{y} \cos \varphi-\dot{x} \sin \varphi)+\frac{1}{2} I \dot{\varphi}^{2}+\frac{1}{2} C\left(\dot{\Psi}_{1}^{2}+\dot{\Psi}_{2}^{2}\right)
$$

(i) and the nonholonomic constraints,

$$
\begin{gathered}
\phi_{1}=\dot{x}+\frac{a \cos \varphi}{2} \dot{\Psi}_{1}+\frac{a \cos \varphi}{2} \dot{\Psi}_{2}, \\
\phi_{2}=\dot{y}+\frac{a \sin \varphi}{2} \dot{\Psi}_{1}+\frac{a \sin \varphi}{2} \dot{\Psi}_{2}, \\
\phi_{3}=\dot{\varphi}+\frac{a}{2 r} \dot{\Psi}_{1}-\frac{a}{2 r} \dot{\Psi}_{2} .
\end{gathered}
$$

These constraints are linear in the velocities and determine a distribution $\mathcal{D}$ on $Q$ whose annihilator is generated by the 1 -forms,

$$
\begin{gathered}
\mu_{1}=d x+\frac{a \cos \varphi}{2} d \Psi_{1}+\frac{a \cos \varphi}{2} d \Psi_{2}, \\
\mu_{2}=d y+\frac{a \sin \varphi}{2} d \Psi_{1}+\frac{a \sin \varphi}{2} d \Psi_{2}, \\
\phi_{3}=d \varphi+\frac{a}{2 r} d \Psi_{1}-\frac{a}{2 r} d \Psi_{2} .
\end{gathered}
$$
Q:

Consider the group of Euclidean motions in the plane, $G=\mathrm{R}^{2} \times S^{1}$, with its standard action on 


$$
\begin{gathered}
\Phi: G \times Q \rightarrow Q, \\
(r, s, \theta) \times\left(x, y, \varphi, \Psi_{1}, \Psi_{2}\right) \mapsto\left(r+x \cos \theta-y \sin \theta, s+x \sin \theta+y \cos \theta, \theta+\varphi, \Psi_{1}, \Psi_{2}\right),
\end{gathered}
$$

whose infinitesimal generators are

$$
\left\langle\frac{\partial}{\partial x}, \frac{\partial}{\partial y},-y \frac{\partial}{\partial x}+x \frac{\partial}{\partial y}+\frac{\partial}{\partial \varphi}\right\rangle .
$$

Observe that the distribution $\mathcal{D}$ is $G$-invariant and, hence, also the constraint submanifold $D$ is preserved, that is, for all $x \in D$ and $g \in G, T \Phi_{g}(x) \in D$.

Taking $\left(x, y, \varphi, \Psi_{1}, \Psi_{2}, \dot{\Psi}_{1}, \dot{\Psi}_{2}\right)$ as coordinates on $D$, we have that the pull-back $\omega_{D}$ of the Poincaré-Cartan 2-form $\omega_{L}$ to $D$ is

$$
\begin{aligned}
\omega_{D}= & \left(-\frac{a m \cos \varphi}{2}+\frac{a m \sin \varphi}{2} \dot{\Psi}_{1}+\frac{a m_{0} l \sin \varphi}{2 r}\right) d x \wedge d \dot{\Psi}_{1} \\
& +\left(-\frac{a m \cos \varphi}{2}+\frac{a m \sin \varphi}{2} \dot{\Psi}_{2}-\frac{a m_{0} l \sin \varphi}{2 r}\right) d x \wedge d \dot{\Psi}_{2} \\
& +\left(-\frac{a m \sin \varphi}{2}-\frac{a m \cos \varphi}{2} \dot{\Psi}_{2}-\frac{a m_{0} l \cos \varphi}{2 r}\right) d y \wedge d \dot{\Psi}_{1} \\
& +\left(-\frac{a m \sin \varphi}{2}-\frac{a m \cos \varphi}{2} \dot{\Psi}_{2}+\frac{a m_{0} l \cos \varphi}{2 r}\right) d y \wedge d \dot{\Psi}_{2} \\
& +\frac{a m_{0} l \sin \varphi}{2 r}\left(\dot{\Psi}_{1}-\dot{\Psi}_{2}\right) d y \wedge d \varphi+\frac{a m_{0} l \cos \varphi}{2 r}\left(\dot{\Psi}_{1}-\dot{\Psi}_{2}\right) d x \wedge d \varphi \\
& -\frac{a I}{2 r} d \varphi \wedge d \dot{\Psi}_{1}+\frac{a I}{2 r} d \varphi \wedge d \dot{\Psi}_{2}+C d \Psi_{1} \wedge d \dot{\Psi}_{1}+C d \Psi_{2} \wedge d \dot{\Psi}_{2} .
\end{aligned}
$$

A basis of $U=\mathcal{D}^{v} \cap T D$ is given by the vectors fields

$$
\begin{gathered}
\left\langle\frac{\partial}{\partial \dot{\Psi}_{1}}, \frac{\partial}{\partial \dot{\Psi}_{2}}, \frac{\partial}{\partial \Psi_{1}}-\frac{a}{2} \cos \varphi \frac{\partial}{\partial x}-\frac{a}{2} \sin \varphi \frac{\partial}{\partial y}-\frac{a}{2 r} \frac{\partial}{\partial \varphi}\right. \\
\left.\quad \frac{\partial}{\partial \Psi_{2}}-\frac{a}{2} \cos \varphi \frac{\partial}{\partial x}-\frac{a}{2} \sin \varphi \frac{\partial}{\partial y}+\frac{a}{2 r} \frac{\partial}{\partial \varphi}\right\rangle
\end{gathered}
$$

Observe again that $D / G$ can be identified with the space $T(Q / G)$. The projected 2-form $\omega_{\bar{U}}^{-}$is

$$
\begin{aligned}
\omega_{U}^{-}= & -\frac{a^{3}}{4 r^{2}} m_{0} l\left(\dot{\Psi}_{1}-\dot{\Psi}_{2}\right) d \Psi_{1} \wedge d \Psi_{2}+\left(\frac{m a^{2}}{4}+\frac{I a^{2}}{4 r^{2}}+C\right) d \Psi_{1} \wedge d \dot{\Psi}_{1} \\
& +\left(\frac{m a^{2}}{4}+\frac{I a^{2}}{4 r^{2}}+C\right) d \Psi_{2} \wedge d \dot{\Psi}_{2}+\left(\frac{m a^{2}}{4}-\frac{I a^{2}}{4 r^{2}}\right) d \Psi_{1} \wedge d \dot{\Psi}_{2} \\
& +\left(\frac{m a^{2}}{4}-\frac{I a^{2}}{4 r^{2}}\right) d \Psi_{2} \wedge d \dot{\Psi}_{1} .
\end{aligned}
$$

This 2 -form $\omega_{\bar{U}}^{-}$is an almost symplectic form, that is, it is nondegenerate but not closed. The solution of the dynamics after the reduction procedure is given by 


$$
i_{\bar{X}} \omega_{\bar{U}}^{-}=d E_{L *}
$$

where $L^{*}: T\left(\mathrm{~T}^{2}\right) \rightarrow \mathrm{R}$ is defined by

$$
L^{*}\left(\Psi_{1}, \Psi_{2}, \dot{\Psi}_{1}, \dot{\Psi}_{2}\right)=\frac{1}{8} m a^{2}\left(\dot{\Psi}_{1}+\dot{\Psi}_{2}\right)^{2}+\frac{I a^{2}}{8 r^{2}}\left(\dot{\Psi}_{2}-\dot{\Psi}_{1}\right)^{2}+\frac{1}{2} C\left(\dot{\Psi}_{1}^{2}+\dot{\Psi}_{2}^{2}\right)
$$

Alternatively, it is possible to find an equation in terms of a symplectic 2-form, but with an additional gyroscopic type 1-form:

$$
i_{X} \omega_{L^{*}}=d E_{L^{*}}+\overline{\alpha_{\Gamma_{L, D}}}
$$

where

$$
\overline{\alpha_{\Gamma_{L, D}}}=\frac{m_{0} l a^{3}}{4 r^{2}}\left(\dot{\Psi}_{2}-\dot{\Psi}_{1}\right) \dot{\Psi}_{2} d \Psi_{1}-\frac{m_{0} l a^{3}}{4 r^{2}}\left(\dot{\Psi}_{2}-\dot{\Psi}_{1}\right) \dot{\Psi}_{1} d \Psi_{2}
$$

\section{ACKNOWLEDGMENTS}

This work was partially supported by grants DGICYT (Spain) PB94-0106, Consejeria de Educacion y Cultura de la Comunidad de Madrid, Universidad de La Laguna and by the Fund for Scientific Research (Flanders, Belgium). D. Martín de Diego also wishes to thank the University of Gent (Belgium) for a Visiting Research Grant.

${ }^{1}$ P. A. M. Dirac, “Lectures on quantum mechanics,” Belfer Graduate School of Science, Yeshiva University, New York, 1964.

${ }^{2}$ M. J. Gotay, "Presymplectic manifolds, geometric constraint theory and the Dirac-Bergmann theory of constraints," Ph.D. thesis, Center for Theoretical Physics, University of Maryland, 1979.

${ }^{3}$ M. J. Gotay and J. M. Nester, "Presymplectic Lagrangian systems I: The constraint algorithm and the equivalence theorem,” Ann. Inst. Henri Poincare, Sect. A 30, 129-142 (1979).

${ }^{4}$ M. J. Gotay and J. M. Nester, "Presymplectic Lagrangian systems II: The second-order differential equation problem," Ann. Inst. Henri Poincaré, Sect. A 32, 1-13 (1980).

${ }^{5}$ G. Marmo, G. Mendella, and W. M. Tulczyjew, "Symmetries and constants of the motion for dynamics in implicit form," Ann. Inst. Henri Poincaré: Phys. Theor. 57, 147-166 (1992).

${ }^{6}$ E. A. Lacomba and W. M. Tulczyjew, "Geometric formulation of mechanical systems with one-sided constraints," J. Phys. A 23, 2801-2813 (1990).

${ }^{7}$ A. Ibort, E. A. Lacomba, M. de León, D. Martín de Diego, and P. Pitanga, “Mechanical systems subjected to impulsive constraints," J. Phys. A 30, 5835-5854 (1997).

${ }^{8}$ J. Neimark and N. Fufaev, "Dynamics of nonholonomic systems,"' Transactions of Mathematical Monographs, Vol. 33 (American Mathematical Society, Providence, 1972).

${ }^{9}$ A. M. Vershik and L. D. Faddeev, "Differential geometry and Lagrangian mechanics with constraints," Sov. Phys. Dokl. 171, 34-36 (1972).

${ }^{10}$ A. M. Vershik, "Classical and non-classical dynamics with constraints," Global Analysis-Studies and Applications I, Lecture Notes in Mathematics No. 1108 (Springer-Verlag, Berlin, 1984), pp. 278-301.

${ }^{11}$ A. Ibort, M. de León, G. Marmo, D. and Martín de Diego, "Non-holonomic constrained systems as implicit differential equations," in Geometrical Structures for Physical Theories, I, Rend. Sem. Mat. Univ. Pol. Torino, Vol. 54, No. 3 (Università e Politecnico, Torino, 1966), pp. 295-317.

${ }^{12}$ J. Koiller, "Reduction of some classical non-holonomic systems with symmetry," Arch. Ration. Mech. Anal. 118, 113-148 (1992).

${ }^{13}$ E. Massa and E. Pagani, "Classical dynamics of non-holonomic systems: A geometric approach," Ann. Inst. Henri Poincaré: Phys. Theor. 55, 511-544 (1991).

${ }^{14}$ W. Sarlet, F. Cantrijn, and D. J. Saunders, "A differential geometric setting for mixed first- and second-order ordinary differential equations,"' J. Phys. A 30, 4031-4052 (1997).

${ }^{15}$ A. M. Bloch, P. S. Krishnaprasad, J. E. Marsden, and R. M. Murray, "Nonholonomic mechanical systems with symmetry," Arch. Ration. Mech. Anal. 136, 21-99 (1996).

${ }^{16}$ A. D. Lewis, “Aspects of geometric mechanics and control of mechanical systems," Ph.D. Thesis, California Institute of Technology, Pasadena, 1995.

${ }^{17}$ L. Bates and J. Śniatycki, “Nonholonomic reduction,’ Rep. Math. Phys. 32, 99-115 (1992).

${ }^{18}$ W. S. Koon and J. E. Marsden, "Poisson reduction of nonholonomic mechanical systems with symmetry," in Proceedings of the Workshop on Non-Holonomic Constraints in Dynamics, Calgary, 26-29 August 1997, Rep. Math. Phys. 42, 101-134 (1998). 
${ }^{19}$ C.-M. Marle, "Reduction of constrained mechanical systems and stability of relative equilibria," Commun. Math. Phys. 174, 295-318 (1995)

${ }^{20}$ M. de León and P. R. Rodrigues, Methods of Differential Geometry in Analytical Mechanics, Math. Ser. 152 (NorthHolland, Amsterdam, 1989).

${ }^{21}$ G. Hinds, "Foliations and the Dirac theory of constraints," Ph.D. thesis, University of Maryland, 1965.

${ }^{22}$ J. F. Cariñena, “Theory of singular Lagrangians,” Fortschr. Phys. 88, 641-679 (1990).

${ }^{23}$ M. Henneaux and C. Teitelboim, Quantization of Gauge Systems (Princeton University Press, Princeton, NJ, 1992).

${ }^{24}$ M. de León and D. Martín de Diego, "On the geometry of non-holonomic Lagrangian systems," J. Math. Phys. 37, 3389-3414 (1996).

${ }^{25}$ A. Ibort, M. de León, J. C. Marrero, and D. Martín de Diego, “Dirac brackets in constrained dynamics,” Fortschr. Phys. (in press, 1998).

${ }^{26}$ M. de León and D. Martín de Diego, "A constraint algorithm for singular Lagrangians subjected to nonholonomic constraints," J. Math. Phys. 38, 3055-3062 (1997).

${ }^{27}$ F. Cantrijn, M. de León, J. C. Marrero, and D. Martín de Diego, "Reduction of nonholonomic mechanical systems with symmetries," in Ref. 18, pp. 25-45.

${ }^{28}$ M. de León, J. C. Marrero, and D. Martín de Diego, "Mechanical systems with non-linear constraints," Int. J. Theor. Phys. 36, 973-989 (1997)

${ }^{29}$ C.-M. Marle, "Various approaches to conservative and nonconservative nonholonomic systems," in Ref. 18, pp. 211229.

${ }^{30}$ A. J. van der Schaft and B. M. Maschke, “'On the Hamiltonian formulation of nonholonomic mechanical systems, ” Rep. Math. Phys. 34, 225-233 (1994).

${ }^{31}$ A. Ibort, M. de León, J. C. Marrero, and D. Martín de Diego, "A Dirac-bracket for non-holonomic Lagrangian systems," in Proceedings of the Vth Fall Workshop: Differential Geometry and its Applications, Jaca, 23-25 September 1996.

${ }^{32}$ F. Cantrijn, M. de León, and D. Martín de Diego, “'On almost-Poisson structures in nonholonomic mechanics,” preprint, 1998.

${ }^{33}$ L. Bates, H. Graumann, and C. MacDonnell, “Examples of gauge conservation laws in nonholonomic systems,” Rep. Math. Phys. 37, 295-308 (1996).

${ }^{34}$ W. S. Koon and J. E. Marsden, "The Hamiltonian and Lagrangian approaches to the dynamics of nonholonomic systems," Rep. Math. Phys. 40, 21-62 (1997).

${ }^{35}$ J. Śniatycki, "Non-holonomic Noether theorem and reduction of symmetries," in Ref. 18, pp. 5-23.

${ }^{36}$ P. Libermann and C.-M. Marle, Symplectic Geometry and Analytical Mechanics (Reidel, Dordrecht, 1987).

${ }^{37}$ R. Abraham and J. E. Marsden, Foundations of Mechanics, 2nd ed. (Benjamin Cummings, Reading, MA, 1978).

${ }^{38}$ J. E. Marsden, R. Montgomery, and T. Ratiu, "Reduction, symmetry, and phases in mechanics," Mem. Am. Math. Soc., Vol. 436 (American Mathematical Society, Providence, 1990).

${ }^{39}$ R. Cushman, D. Kemppainen, J. Śniatycki, and L. Bates, “Geometry of nonholonomic constraints,' Rep. Math. Phys. 36, 275-286 (1995).

${ }^{40}$ F. Cantrijn, M. de León, and D. Martín de Diego, "The momentum equation for non-holonomic systems with symmetry," in Proceedings of the National Conference on Theoretical and Applied Mechanics, Leuven, 22-23 May 1997, pp. $31-34$.

${ }^{41}$ A. Capri and M. Kobayashi, “A mechanical model with constraints,” J. Math. Phys. 23, 736-741 (1982).

${ }^{42}$ A. Capri and M. Kobayashi, "The first-rank tensor field coupled to an electromagnetic field," J. Phys. A 26, 6101-6112 (1987).

${ }^{43}$ A. Echeverría-Enríquez, M. C. Muñoz-Lecanda, and N. Román-Roy, "Reduction of presymplectic manifolds with symmetry,"' preprint, 1997. 